\title{
Tissue Integration and Biological Cellular Response of SLM-Manufactured Titanium Scaffolds
}

\author{
Anida-Maria Băbțan ${ }^{1, \dagger}{ }^{+}$Daniela Timuș ${ }^{2,+}$, Olga Sorițău ${ }^{3}$, Bianca Adina Boșca ${ }^{4, *} \mathbb{1}$, \\ Reka Barabas ${ }^{5}$, Anca Ionel ${ }^{1}$, Nausica Bianca Petrescu ${ }^{1}$, Claudia Nicoleta Feurdean ${ }^{1}$, \\ Ioana Roxana Bordea ${ }^{6}$, George Saraci ${ }^{7, *}$, Ştefan Cristian Vesa ${ }^{8}$ (D) and Aranka Ilea ${ }^{1}$ (D) \\ 1 Department of Oral Rehabilitation, Faculty of Dentistry, Iuliu Hațieganu University of Medicine and \\ Pharmacy, 400012 Cluj-Napoca, Romania; Babtan.Anida@umfcluj.ro (A.-M.B.); anca_ionel@yahoo.com (A.I.); \\ nausica_petrescu@yahoo.com (N.B.P.); cbraitoru@yahoo.com (C.N.F.); arankailea@yahoo.com (A.I.) \\ 2 Faculty of Dentistry, Iuliu Hațieganu University of Medicine and Pharmacy, 400012 Cluj-Napoca, Romania; \\ timus.daniela.g@gmail.com \\ 3 Laboratory of Radiotherapy, Tumour and Radiobiology, Prof. Dr. Ion Chiricuță Oncological Institute, \\ 400015 Cluj-Napoca, Romania; olgasoritau@yahoo.com \\ 4 Department of Histology, Faculty of Medicine, Iuliu Hațieganu University of Medicine and Pharmacy, \\ 400349 Cluj-Napoca, Romania \\ 5 Department of Chemistry and Chemical Engineering, Faculty of Chemistry and Chemical Engineering, \\ Babeș-Bolyai University, 400028 Cluj-Napoca, Romania; reka.barabas@gmail.com \\ 6 Department of Oral Health, Faculty of Dentistry, Iuliu Hatieganu University of Medicine and Pharmacy, \\ 400012 Cluj-Napoca, Romania; roxana.bordea@ymail.com \\ 7 Graduate of Iuliu Haţieganu Faculty of Medicine, University of Medicine and Pharmacy, \\ 400337 Cluj-Napoca, Romania \\ 8 Department of Pharmacology, Toxicology, and Clinical Pharmacology, Faculty of Medicine, Iuliu Hațieganu \\ University of Medicine and Pharmacy, 400337 Cluj-Napoca, Romania; stefanvesa@gmail.com \\ * Correspondence: biancabosca@yahoo.com (B.A.B.); gsaraci@yahoo.com (G.S.) \\ + Anida-Maria Băbțan and Daniela Timus have equal contribution as first authors of this article.
}

Received: 5 August 2020; Accepted: 2 September 2020; Published: 5 September 2020

Abstract: Background: SLM (Selective Laser Melting)-manufactured Titanium (Ti) scaffolds have a significant value for bone reconstructions in the oral and maxillofacial surgery field. While their mechanical properties and biocompatibility have been analysed, there is still no adequate information regarding tissue integration. Therefore, the aim of this study is a comprehensive systematic assessment of the essential parameters (porosity, pore dimension, surface treatment, shape) required to provide the long-term performance of Ti SLM medical implants. Materials and methods: A systematic literature search was conducted via electronic databases PubMed, Medline and Cochrane, using a selection of relevant search MeSH terms. The literature review was conducted using the preferred reporting items for systematic reviews and meta-analysis (PRISMA). Results: Within the total of 11 in vitro design studies, 9 in vivo studies, and 4 that had both in vitro and in vivo designs, the results indicated that SLM-generated Ti scaffolds presented no cytotoxicity, their tissue integration being assured by pore dimensions of 400 to $600 \mu \mathrm{m}$, high porosity (75-88\%), hydroxyapatite or $\mathrm{SiO}_{2}-\mathrm{TiO}_{2}$ coating, and bioactive treatment. The shape of the scaffold did not seem to have significant importance. Conclusions: The SLM technique used to fabricate the implants offers exceptional control over the structure of the base. It is anticipated that with this technique, and a better understanding of the physical interaction between the scaffold and bone tissue, porous bases can be tailored to optimize the graft's integrative and mechanical properties in order to obtain structures able to sustain osseous tissue on Ti.

Keywords: titanium; selective laser melting; mesenchymal stem cell; hydroxyapatite; scaffolds 


\section{Introduction}

Background

Biomedical applications are an important direction for 3D (three-dimensional) printing, in particular for tissue and function reconstruction by scaffolds. Use of 3D printing allows the personalization of implants, so that they will be suitable for the recipient area. Receptor situs can be scanned for an optimal adaptation. 3D print has the advantage to reduce material consumption in comparison to conventional substractive scaffold technologies. This could bring a financial equilibrium for the equipment for an extended time period [1]. Hutmacher et al. defined the features of bone tissue biological scaffold that can be assigned to dental implants: (1) biocompatibility, which promotes the cell proliferation; (2) corresponding mechanical features to the implants' receptor situs; (3) a degree of porosity that allows interior cell proliferation, and transport of nutritive substances and unused material; (4) favourable microsurface for cellular adsorption and regeneration [2].

Scaffolds' biological integration is related to the manufacture process and specific material. Titanium (Ti) is known to have excellent biocompatibility. Moreover, it is important for a biomedical device to have similar mechanical properties to the bone tissue, in order to enhance its purpose for the human body. The lack of these characteristics induces bone loss and mobilization of implants [3], thus affecting the bioimplants' maintenance [4]. An alternative would be the use of Ti pore and cell scaffolds, which are manufactured to possess mechanic features close to the hard biological tissues [5]. Porous structures are used due to their anatomic similarity to trabecular bone, and they allow cellular ingrowth, which has not been confirmed yet by clinical research for non-porous scaffolds $[6,7]$. In addition, implants' microsurface influences the behaviour of osteoblasts and osteocytes and promotes new bone formation [8].

Laser intensity has a strong influence on materials' structure and properties: The higher the power and decreased scanning speed, the lower the surfaces' porosity, but it also increases the materials' texture and its mechanical properties anisotropy [9]. The delivered energy varies during the manufacture process and influences the chemical composition of the surface of unprocessed powder (Ti oxide layer), which decreases (up to $60 \%$ ) after thermal and chemical treatment; moreover, chemical treatment (19\% hydrofluoric acid and $8 \%$ nitric acid) can cause a decrease by $2-6$ times of Ti surface energy and an increase up to fivefold times for compressive stress [10].

Cellular adhesion (fibronectin, mesenchymal stem cells, collagen fibers) is related to the porosity degree of Ti scaffold surface; improved adhesion was observed on rough scaffolds, with positive surface charge and moderate hydrophilicity [11,12]. Cell metabolic activity could be influenced by the degree of adhesion, by the removal of unmelted Ti powder, which leads to a smoother surface, increased surface energy and consequently proliferation medium [9]. The type of Ti scaffold alloy is associated to cellular behaviour. $\mathrm{Ti}_{6} \mathrm{Al}_{4} \mathrm{~V}-6 \mathrm{Cu}$ selective laser melting (SLM) alloys could inhibit the activity of proinflammatory cytokines, upregulate angiogenesis and $\mathrm{Ni}-\mathrm{Cr}$ SLM alloys present a lower human adipose stem cells proliferation and viability compared to $\mathrm{Co}-\mathrm{Cr}[13,14]$. The addition of Hydroxyapatyte (HA) or bioactive ceramics, such as $\beta$-tricalcium phosphate to Ti SLM scaffolds, could allow cellular attachment and multiplication on and inside the scaffolds' structure, and by its influence on $\mathrm{pH}$ value, could improve cell metabolism $[15,16]$. Stiffness, pore size, surface roughness, and elasticity have a direct influence on bone regeneration and implant integration. Moderate elasticity and surface roughness $(2-4 \mu \mathrm{m})$ reduce osteoclasts activity and promote earlier healing; elasticity, architectural similarity to cancellous bone and pore dimension range between 450-650 $\mu \mathrm{m}$ are needed in order to ensure interconnectivity and angiogenic and metabolic activity $[17,18]$. Ran et al. evaluated the implications of porosity and pore size of $\mathrm{Ti}_{6} \mathrm{Al}_{4} \mathrm{~V}$ SLM scaffolds in vivo and in vitro [19]. They found that interconnected $600 \mu \mathrm{m}$ pore dimension promotes cell migration, proliferation, differentiation, and biological fluid exchange, without affecting the scaffolds' mechanical properties. Pore dimension was also considered for the dental area and compared to dentine and cementum Young's modulus, which is known to be around 20-25 GPa and proximate to cancellous bone modulus; a dense core 
associated to peripheral larger pores supports cellular proliferation and mechanical resistance [20]. Pure Ti, Ti and alloys were employed in general medicine, dentistry and head and neck surgery for tissue and function substitute, due to their increased resistance to corrosion, significant strength, and tissue and chemical compatibility [21]. In order to achieve biofunction and biocompatibility (molecule adsorption and adhesion, cell activation, tissue proliferation), Ti scaffolds are considered to be the gold standard, due to their osteointegration properties: corrosion resistance, surface hydroxyl groups (which increase the wettability and promote integrin, proteins adsorption), electrostatic forces, and formation of calcium phosphates (due to the continuous partial dissolution and precipitation of outer scaffolds' surface) [22]. In tissue engineering, there are techniques that have the purpose to promote bone formation, soft tissue adhesion, antibacterial properties, where wet and dry scaffolds' surface processes are involved, such as $\mathrm{Ca}, \mathrm{N}, \mathrm{He}$ ion implantation, $\mathrm{HA}, \mathrm{TiO}_{2}$ spray or electrochemical coating, chemical acid etching, alkaline immersion, protein immobilization [23]. All of these practices enhance the artificial grafts integration and biological potential. Studies of Smargiassi and Ding showed that surface scaffold treatment and microirregularities promote cell colonization and osteocyte-like dendritic cells formation, which are associated to a superior osteointegration and osteoconductive properties [24,25].

Regarding the implications of Ti SLM products in reconstruction of bone defects (10-20 mm), the studies of Hirota and Ma evaluated the in vitro (human osteoblasts) and in vivo (rabbit model) biological response induced by Ti porous scaffolds [26,27], used as an alternative to autogenous tibial/femoral graft, which implies multiple surgeries and a high morbidity rate. Their results showed that although $\mathrm{Ti}$ has an elasticity modulus up to 10 times higher compared to trabecular bone, 3D printing allows the manufacture of patient-specific implant scaffold, which by the degree of porosity (60-70\% approaches the natural bone modulus 10-12 GPa) and internal lattice structure enhances the osteointegration and osteogenesis process, despite immediate loading and function, with a resistance force of approximately $200 \mathrm{~N}$. The authors suggested that mechanical stress provided a balanced environment for osteogenic cells, enabling continuous regeneration and remodelling.

Ghosh et al. conducted an experimental study in order to obtain SLM-printed polymer grafted $\mathrm{Ti}_{6} \mathrm{Al}_{4} \mathrm{~V}$ hip prostheses, to counterbalance the surface roughness of untreated Ti known to favour protein adsorption, which is favourable in dental implant osteointegration, but not in hip reconstruction [28]. They found that hydrated methacryloexyethylphosphorylcholine coating layer protects Ti scaffold from friction and hydroxyl ions exchange, minimising surface wear and unfavourable protein adsorption. Besides hip reconstruction, SLM Ti scaffolds find their utility in vertebral reconstruction; Wang et al. produced a Tantalum (Ta)-coated porous $\mathrm{T}_{6} \mathrm{Al}_{4} \mathrm{~V}$ scaffold, which proved to be superior to other coating materials, due to its high strength, corrosion resistance in acidic environment and does not change in weight or surface roughness, and heat resistance [29]. Crovace et al. conducted an animal study, in which a 5 -cm bone fragment on tibial diaphysis of ovines $(\mathrm{N}=12)$ was replaced by an Electron Beam Melting or porous HA Ti scaffold, with a mean pore dimension of $1.5 \mathrm{~mm}$ length $\times 2.4 \mathrm{~mm}$ width [30]. The healing of the tibial bone (after 12 months) resulted in new cortical bone that bridged the defect and continued with lamellar bone in apposition to the titanium pores of the scaffolds.

Head and neck cancer affects up to 60,000 patients in Europe, from which 5-7\% involve oral cavity and jawbones; due to immunogenic reactions of allografts and xenografts, 3D printed implants are considered, along with an associate bioactive implants' surface, such as $\beta$-tricalcium phosphate, HA, bone morphogenic proteins, to enhance the osteoinductive and osteoconductive process [31]. Interestingly, Zhang showed on a rabbit model that nano-HA Ti scaffolds inhibited the close to defect intramuscular-induced tumours [32]. Moreover, the presence of HA in the scaffolds' pores inhibited the tumoral cells into the grafting material. The authors suggested that along with the activated mitochondrial apoptosis pathway, HA upregulates Tumour Necrose Factor Family, which can trigger cell death through an extrinsic pathway-the inflammatory cytokines.

Systematic reviews evaluated 3D printing in reconstruction of maxillo-facial defects [31,33]. Although most of the studies included in the reviews were conducted on animal models, 
the results evidenced the advantages of SLM printing: accurate reconstruction, low morbidity, complex design, improved life quality due to avoidance of flap harvest, aesthetic implications such as lower border contour/temporomandibular joint reconstruction, biological safety (non-cytotoxic), and improved osteointegration.

Even though Ti metal and Ti alloys are biocompatible, there is no direct connection between the scaffold and bone defect. On the other hand, Ti links to bone tissue after either chemical or thermal processing, by a thin apatite microsurface layer; this layer stimulates osteoblasts and osteocytes attachment, multiplication, differentiation, and consequent bone formation [34-36]. Some of these methods are HAP (hydroxyapatite) coating [37,38] or surface treating with heat [39] and $\mathrm{NaCl}[40]$. $\mathrm{Ti}$ alloys have an increased corrosion resistance due to a protective oxide layer, which acts as an inhibitor for ions release [41]. While Ti biomaterials have the disposition to release ions from uncoated surfaces, the application of hydrofluoric acid (HF) could repel the oxidation process and Ti ions loss [42]. This corrosion process of Ti biomaterials can be decreased by adding a titanium nitride coating [43]. It has been stated that a low nitric acid and a high hydrofluoric acid concentration lead to a decreased Ti surface roughness and an increased meltage [44]. Regarding surrounding tissue proliferation, acid surface treatment such as sulfuric, hydrochloric, and nitric solutions does not inhibit cell proliferation or collagen fibers production [45]. Ti scaffolds subjected to HAP processing showed the production of a bone-like apatite stratum, to exhibit osteoconductivity and osteoinductivity [46] and to enhance osteoblast differentiation [47]. Porous Ti scaffolds surface treatment enables cellular attachment, regeneration, promotes the biomaterials' scaled topographical features, and advances the biomaterials' osteointegration due to the apatite layer [48].

The scaffolds' pore architecture provides a microsurface favourable to cellular adsorption and regeneration, which leads to a firm and fix implant-tissue contact. Other improvements could include MSCs (adult mesenchymal stem cells) coating before the implantation. This pre-treatment could enable isolated cells to differentiate into specific bone cells or gingival cells, tissues under the influence of appropriate stimuli [49]. It has been shown that the MSCs loaded on porous scaffolds support the regeneration of bone defects [50].

One of the most promising additive technologies is the SLM (Selective Laser Melting) method. Its set-up includes a laser power supply, powder holders, delivering and layering devices, manufacture surface, and control constants' processing computing device [51]. The manufacture is usually thermoconducted to a constant temperature less than $\sim 20{ }^{\circ} \mathrm{C}$ for the entire $3 \mathrm{D}$ processing. Chosen materials (powdered metals/alloys) are loaded to storage containers. Powders' dimension usually varies from $20 \mu \mathrm{m}$ to $60 \mu \mathrm{m}$; the powder is brought to the production surface and transformed into $30-100 \mu \mathrm{m}$ thickness layers [52].

Other well-known additive technologies in metal manufacture are represented by: Electron Beam Melting (EBM), binder jetting (BJG), and metal extrusion; EBM involves a high temperature $\left(596^{\circ} \mathrm{C}\right)$ process, which results in a less finer microstructure compared to SLM [53]. Moreover, the products obtained using SLM have superior mechanical, corrosion and tribological properties (wear, friction, lubrication) when compared to EBM [54]. Meanwhile, EBM has limitations in the support material (second degree $\mathrm{Ti}, \mathrm{CoCrMo} \mathrm{Ti}_{6} \mathrm{Al}_{4} \mathrm{~V}$, Inconel 718); $\mathrm{SLM}$ is expanded to aluminium, Ti, iron, nickel, cobalt, and copper-based alloys, and because of the cooling process during the manufacturing and variation of several parameters, the mechanical properties of the final product can be induced [55], according to the purpose of the achieved parts. The SLM advantages, modulation of properties, functionality, and large materials options, the soft external microstructure with a complex internal architecture overcomes the needs of Ti-based scaffolds, used in maxillo-facial area reconstruction and dental implant design and osteointegration, which make SLM a focus additive technology for this review.

From a comparative view, in contrast to EBM either laser metal deposition (LMD), SLM has the lowest time product $(80 \mathrm{~min})$, smoother vertical surface, minimal residual stress, no required thermal stress relieve and the largest powder size particle's option and also the lowest elastic modulus 
(3.7-6.7 GPa) [56], which are significant benefits in biomedical technology. In comparison to other mechanical manufacture methods (cast, forged), SLM allows to obtain small support bars 3D NiTi filigree structures and open pores; and the optimization of mechanical parameters is possible by adjusting strut dimension, porosity, pore size, and lattice geometry [57]. The addition of martensite and austenite crystalline structures, which have low Young modulus, enhances the biomimetic shock-absorbing and pseudoelastic properties of SLM scaffolds [58]. Vaithilingam et al. compared $\mathrm{SLM} \mathrm{Ti}_{6} \mathrm{Al}_{4} \mathrm{~V}$ scaffolds with conventional forged samples [59]. Their results showed a lower surface ratio Ti:Al for SLM samples (2.68) compared to conventional ones (4.4), whereas for the surface ions, there was $\mathrm{Al}$, Ti and $\mathrm{V}$ (non-biocompatible) for conventional scaffolds compared to SLM. Regarding biological response, $\mathrm{Ti}_{6} \mathrm{Al}_{4} \mathrm{~V}$ SLM porous scaffolds showed a higher osteogenesis and osteointegration compared to EBM porous scaffolds, which enhanced the healing and proliferation of soft tissue [60]. These results might be related to the surface roughness, being stated that if it has a value lower than $24.9 \mu \mathrm{m}$, it will have a beneficial effect on osteointegration and proliferation [61]. Some SLM disadvantages are the chip formation, powder particles which remains at the surface and the staircase effect due to the increased layer thickness; to overcome these limitations, laser ablation could be associated after SLM, a high energy density of laser beams leading to a decreased surface roughness and reduced capillary waves [57,62].

Besides Ti alloys, other materials are used in biomedical engineering, such as $\mathrm{NiCr}, \mathrm{CoCr}$ and NiTi. SLM NiTi alloys are shape-memory alloys (SMA) (given the transformation of austenite state-stable after thermotreatment and martensitic state-stable at low temperature, allows the redimension after insertion in the receptor situs), resistant to corrosion, and possess non-ferromagnetic properties, which indicate them in bone implants, orthodontic arch wires, palatal expander, distracters, and dental drills [62]. This shape memory property refers to NiTi, $\beta$ type Ni-free Ti materials and their ability to recover its shape after a heating process, due to the pseudoplastic deformation; whereas both non-deformed martensite and deformed martensite can be displayed at $23-24{ }^{\circ} \mathrm{C}$ temperature, the austenite phase is recorded at high temperature, meaning that the alloy can remember its initial morphology after it has been overheated $[63,64]$. At body's temperature, NiTi SLM porous plates are in the martensite phase and require little effort in inserting into the reconstruction area; maintenance of a constant temperature leads to austenite phase transformation, which produces a steady stress to compress the bone fragments [65]. Their compression properties are similar to NiTi produced by conventional methods, but the tensile properties are susceptible to microcracks and impurities; the optimization of SLM parameters upregulates their shape-memory and superelastic properties $[66,67]$. Along with SLM, NiTi and SMA were manufactured by several technologies, such as vacuum sintering, self-propagating high temperature synthesis (SHS), and capsule-free hot isostatic pressing (CF-HIP), which allow large variation of pore size and porosity, to cover the cortical bone (4-30\%) and trabecular bone (up to $90 \%$, using SHS, CF-HIP) [68]. Still, the high degree of porosity negatively influences the compressive strength and elastic modulus, and it has been stated that in order to achieve superior superelastic strain $(>4 \%)$ and strength, NiTi alloys should have a radial gradient porosity design (external $20 \%$ porosity to internal $61 \%$ porosity) [69]. This association could promote scaffold cell proliferation and superior strength.

Although $\mathrm{Ni}$ is known to induce allergies, the $\mathrm{Ni}$ ions released during the corrosion process does not imperative depend on the $\mathrm{Ni} \%$ in the alloy, and that coating technologies (passivation, ageing, electropolishing) positively reduce Ni release [70]. Also, the biocompatibility of these memory-alloys is given by the stable Ti oxide layer formed at the surface of the scaffold after the initial Ti release; also, the degree of porosity and elastic behaviour does not influence the cellular proliferation for human osteoblasts, although central pores stimulate adaptive mechanisms of osteoblasts to change their morphologic aspects [71,72]. Their mechanical and biological properties make them competitive with $\mathrm{Ti}_{6} \mathrm{Al}_{4} \mathrm{~V}$ alloys. NiTi SMA have been proposed for light-weight-actuator, energy absorption, and biomedical field (bone fixation plates and screws, dental root replacement, cervical area bone substitute, vertebral implantation-SHS technique, acetabular cup in hip prostheses); regarding 
dental implants, SMA can be preoperatory deformed and inserted, afterwards the firm fixation being performed at body temperature due to the shape memory effect $[73,74]$. These properties could be associated to surface acid-etching, sandblasting, air-plasma treatment, growth-factors, and micro-arc oxidation, in order to stimulate the osteoblasts' proliferation and osteointegration $[75,76]$. In our opinion, the employment of NiTi SMA in dental implantology could lead to a more predictable lifespan of the overdentures, considering the superelastic property, which could mimic physiological dynamic action of periodontal ligaments. Moreover, these alloys find their relevance in rotary endodontic instruments, where besides NiTi SMA, thermomechanically treated Controlled Memory (CM) Wire, Electrical discharge machining (EDM), Gold/Blue treated files, or maxWire (Martensite-Austenite-electropolish-fileX), which have enhanced fatigue resistance and flexibility compared to conventional NiTi instruments [77-79]. Despite the publication of multiple articles exploring the properties and biocompatibility of the SLM-manufactured scaffolds, there is still no adequate information on the integration of the Ti scaffolds in different tissues. While other reviews explored the mechanical characteristics, the fabrication process or the design of these scaffolds $[80,81]$, none of them presented a detailed and profound analysis of the tissue integration.

To better appreciate this potential, we performed a descriptive review of the previously published studies to collect all available data on the integration of the SLM Ti scaffolds, taking into consideration in vitro and in vivo studies. Thus, this article summarizes through a review of the literature, the essential parameters (porosity, pore dimension, pore geometry, surface treatment, shape) required to assess the long-term performance of Ti SLM scaffolds, allowing to determine the necessity and to reason for future clinical studies references.

\section{Materials and Methods}

This literature review was conducted using the preferred reporting items for systematic reviews and meta-analysis (PRISMA) [82].

\subsection{Eligibility Criteria}

We searched for original articles in English, from their dates of inception up to 1st September 2018. Brief reports, forums, technical notes, abstracts, opinion articles, and reviews were excluded.

\subsection{Information Sources}

Information sources included PubMED, MEDLINE, and the COCHRANE Library, with published articles only being selected.

\subsection{Search Strategy}

Medical Subject Headings (MeSH) were used for electronic database searches. For each search strategy, a thorough list of synonyms was used, combined with the conjunction "OR". After that, the searches designated according to the main aspects were combined with "AND" in order to get the list of publications from which the relevant ones were picked. The primary searches were conducted in English, using the following MeSH terms and search term combinations:

"Selective laser melting" [MeSH term] AND "titanium", "selective laser melting" [MeSH term] AND "dentistry", "selective laser melting" [MeSH term] AND “dentistry" AND "titanium", "selective laser melting" [MeSH term] AND "orthopedic", "selective laser melting" [MeSH term] AND "orthopedic" AND "titanium", "selective laser melting" [MeSH term] AND "stem cells", "selective laser melting" [MeSH term] AND "stem cells" AND "dentistry", "selective laser melting" [MeSH term] AND "scaffolds", "selective laser melting" [MeSH term] AND "scaffolds" AND "titanium", "selective laser melting" [MeSH term] AND "tissue engineering". 


\subsection{Study Selection}

Two examiners read the titles and abstracts of all studies, and no blinding was carried out regarding author names, journal or publication date. All articles deemed potentially eligible for inclusion were retrieved in full-text format. Studies published only in abstract form were excluded. The search was completed with a review of the references of the selected articles in order to identify additional studies not found in the initial literature search. Disagreements on study inclusion or endpoints were resolved by a third author.

\subsection{Eligible Studies}

(1) Inclusion criteria

Before starting the study, a series of inclusion and exclusion criteria were established. Chosen full-text articles were assessed for the following inclusion criteria: (a) Prospective and retrospective studies, case series; (b) In vitro or animal studies; (c) IMRAD (Introduction, Methods, Results, and Discussion) structure; (d) Articles from the last 20 years; (e) Presence of references; (f) 1 week (for the in vivo studies) and 1 day (for the in vitro studies) follow-up at least.

\section{(2) Exclusion criteria}

Published studies not meeting the inclusion criteria were excluded from this systematic review (i.e., publications in languages others than English, educational statements, expert opinions, narrative reviews on the subject). The following exclusion criteria were applied: (a) Absence of abstract; (b) Absence of full-text; (c) Duplicated studies; and (d) Case reports or systematic reviews. Publications were not included if they did not provide SLM-related data concerning its biocompatibility, if they did not have an experimental part, or if they did not provide any information concerning the focused question.

\subsection{Data Extaraction and Synthesis of Results}

Two reviewers independently extracted data from each article included. Relevant data from all included articles was summarized in evidence tables. Evidence tables contained the following information: study design, scaffold type, porosity, pore dimension, material, surface treatment, follow-up, and number of probes. The evidence tables were pilot-tested.

\section{Results}

\subsection{Study Selection}

Our search in databases identified 234 articles with the queried keywords, all of them in MEDLINE (PubMed), and none selected from Cochrane. We excluded 27 articles because they were not written in English, and another 34 studies because they were not full-text available, resulting in 173 articles. These were independently read in full by two of the authors who appraised their suitability for inclusion in the study. A total of 94 studies were excluded because they did not fulfill the eligibility criteria, and 51 were excluded because they contained excluding criteria. Finally, 29 studies on the integration of the SLM fabricated scaffolds were selected for this systematic review. A flowchart of the entire process of review can be seen in Figure 1.

\subsection{Study Characteristics}

The origin of studies by country was: United States $(n=1)$, The Netherlands $(n=4)$, Switzerland $(n=1)$, Germany $(n=4)$, Canada $(n=1)$, China $(n=3)$, Japan $(n=4)$, Romania $(n=2)$, Poland $(n=1)$, Czech Republic $(n=1)$, United Kingdom $(n=2)$, Belgium $(n=1)$, Spain $(n=1)$, China $(n=2)$, and Italy $(n=1)$. Publication dates ranged from 2009 to 2017, and all selected articles were written in English. 


\subsection{Overall Characteristics of the Included Studies}

The 24 studies that met the inclusion criteria are summarized in Table 1.

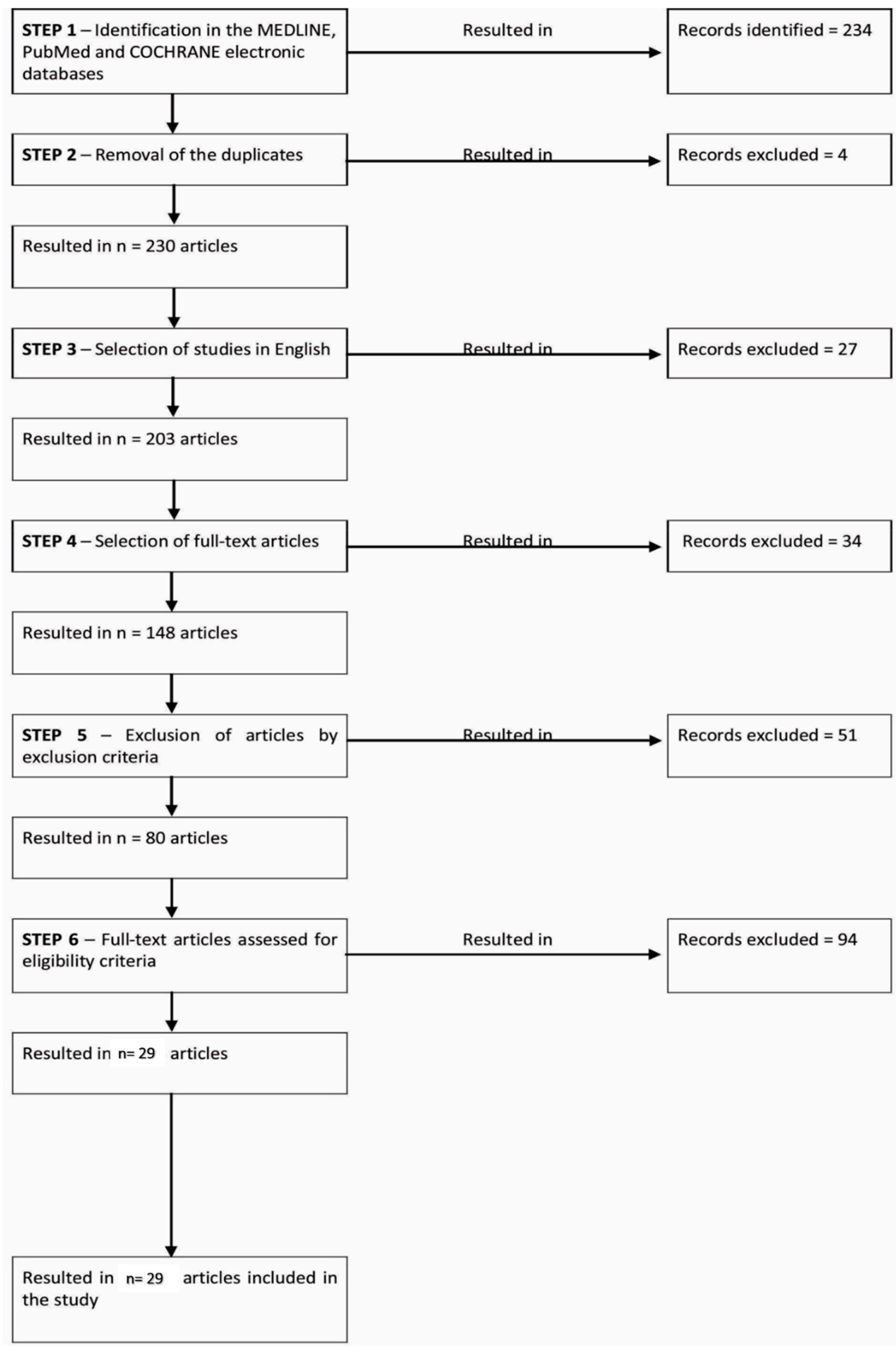

Figure 1. Study screening and selection process. 
Table 1. Detailed data of the included studies.

\begin{tabular}{|c|c|c|c|c|c|c|c|c|}
\hline $\begin{array}{l}\text { Author, Year, } \\
\text { Reference }\end{array}$ & Study Design & $\begin{array}{l}\text { Scaffold Size, } \\
\text { Dimension }\end{array}$ & $\begin{array}{c}\text { Pore Size, } \\
\text { Architecture }\end{array}$ & Porosity & Material & $\begin{array}{c}\text { Surface } \\
\text { Treatment }\end{array}$ & Number of Probes & Follow-Up \\
\hline $\begin{array}{l}\text { Bartolomeu et al., } \\
\quad 2020[17]\end{array}$ & in vitro & $\begin{array}{c}\text { Ø } 4.3 \mathrm{~mm} \times \text { height } \\
4.8 \mathrm{~mm} \times 5.6\end{array}$ & $500,600 \mu \mathrm{m}$ & $64-93 \%$ & $\mathrm{Ti}_{6} \mathrm{Al}_{4} \mathrm{~V}$ & - & $\begin{array}{l}\text { L929 murine } \\
\text { fibroblast cell line }\end{array}$ & $1,7,14,21,28$ days \\
\hline $\begin{array}{l}\text { Bartolomeu et al., } \\
2020 \text { [17] }\end{array}$ & in vitro & $\begin{array}{c}\varnothing 4.3 \mathrm{~mm} \times \text { height } \\
4.8 \mathrm{~mm} \times 5.6\end{array}$ & $500,600 \mu \mathrm{m}$ & $64-93 \%$ & $\mathrm{Ti}_{6} \mathrm{Al}_{4} \mathrm{~V}$ & - & $\begin{array}{l}\text { L929 murine } \\
\text { fibroblast cell line }\end{array}$ & $1,7,14,21,28$ days \\
\hline Liu et al., 2020 [18] & in vitro & $\begin{array}{l}\varnothing 10 \mathrm{~mm} \text { height } \times \\
20 \mathrm{~mm}, \varnothing 5 \mathrm{~mm} \\
\text { height } \times 15 \mathrm{~mm}\end{array}$ & Gyroid, diamond & - & $\mathrm{Ti}_{6} \mathrm{Al}_{4} \mathrm{~V}$ & - & Primary osteoblasts & 7 days \\
\hline $\begin{array}{c}\text { Hanawa et al., } 2020 \\
\text { [22] }\end{array}$ & in vitro & $\begin{array}{l}\varnothing 6.76 \text { height } \times \\
4.33 \mathrm{~mm}\end{array}$ & $400-650 \mu \mathrm{m}$ & - & $\mathrm{Ti}_{6} \mathrm{Al}_{4} \mathrm{~V}$ & - & Primary osteoblasts & $1,7,14,21,28$ days \\
\hline $\begin{array}{c}\text { Crovace et al., } 2020 \\
\text { [30] }\end{array}$ & in vivo & $\begin{array}{c}\varnothing 40 \mathrm{~cm} \times 12 \mathrm{~mm} \\
\text { diameter }\end{array}$ & 1.5-2.4 mm, Gyroid & - & $\mathrm{Ti}_{6} \mathrm{Al}_{4} \mathrm{~V}$ & - & 12 sheep & 1 year \\
\hline $\begin{array}{c}\text { Zhang et al., } 2019 \\
\text { [32] }\end{array}$ & in vitro in vivo & - & $504 \mu \mathrm{m}$ & $65 \%$ & $\mathrm{Ti}_{6} \mathrm{Al}_{4} \mathrm{~V}$ & - & $\begin{array}{l}\text { VX2 tumor cells, L929 } \\
\text { murine cells, Rabbits }\end{array}$ & $1,3,5$ days, 5 weeks \\
\hline $\begin{array}{c}\text { Strauss et al., } 2012 \\
\text { [83] }\end{array}$ & in vitro & $\begin{array}{c}\text { Ø } 2.5 \mathrm{~mm} \times \text { height } \\
10 \mathrm{~mm}\end{array}$ & - & - & TiNi (Nitinol) & - & $\begin{array}{l}10^{5} \text { human ASCs } \\
\text { from fat tissue/well }\end{array}$ & 24 h, 48 h, 6 weeks \\
\hline $\begin{array}{c}\text { Tsukanaka et al., } \\
2016 \text { [84] }\end{array}$ & in vitro & $\begin{array}{c}\varnothing 1 \mathrm{~mm} \times \text { height } \\
14 \mathrm{~mm} \times \text { thickness } \\
14 \mathrm{~mm}\end{array}$ & 20-180 nm, Plate & - & CpTi & $\begin{array}{l}\text { diluted } \mathrm{HCl} \text {, } \\
\text { alkali and heat }\end{array}$ & $\begin{array}{c}10^{5} \text { osteoblast from } \\
\text { neonatal mouse } \\
\text { calvaria/12 scaffolds }\end{array}$ & $2,7,14$ days \\
\hline $\begin{array}{c}\text { Amin Yavari et al., } \\
2015 \text { [85] }\end{array}$ & in vitro & $\begin{array}{c}\text { disk: } \varnothing 8 \mathrm{~mm} \times \\
\text { height } 3 \mathrm{~mm}\end{array}$ & $500 \mu \mathrm{m}$, Disk & - & $\mathrm{Ti}_{6} \mathrm{Al}_{4} \mathrm{~V}$ & $500^{\circ} \mathrm{C}$ heat & 50,000 hPDC/scaffold & 21 days \\
\hline $\begin{array}{c}\text { Markhoff et al., } 2015 \\
\text { [86] }\end{array}$ & in vitro & - & $\begin{array}{c}\text { C: } 700 \mu \mathrm{m}, \mathrm{P}: \\
400-620 \mu \mathrm{m}, \mathrm{D}: \\
400-1000 \mu \mathrm{m}, \text { cubic, } \\
\text { pyramidal, diagonal }\end{array}$ & $\begin{array}{l}\text { C: } 51 \%, \text { P: } \\
76 \%, \text { D: } 75 \%\end{array}$ & Ti-6Al-4V & - & $\begin{array}{c}4 \times 10^{5} \text { human, } \\
\text { osteoblasts/scaffold }\end{array}$ & $1,4,8$ days \\
\hline $\begin{array}{c}\text { Nover et al., } 2015 \\
\text { [87] }\end{array}$ & in vitro & $\begin{array}{l}\varnothing 4 \text { and } 10 \mathrm{~mm} \times \\
\text { height } 7 \mathrm{~mm}\end{array}$ & $\begin{array}{c}\text { 600, 900, and } 1200 \\
\mu \mathrm{m}, \text { Cylindrical }\end{array}$ & - & $\begin{array}{l}\text { commercially } \\
\text { pure Ti }\end{array}$ & - & $\begin{array}{l}30 \times 10^{6} \text { articular } \\
\text { cartilage harvested } \\
\text { from adult canine } \\
\text { knees cells } / \mathrm{mL}\end{array}$ & $14,28,35$ days \\
\hline $\begin{array}{c}\text { Wysocki et al., } 2016 \\
\text { [88] }\end{array}$ & in vitro & $\begin{array}{c}\varnothing 6 \mathrm{~mm} \times \text { height } \\
4 \mathrm{~mm}\end{array}$ & $\begin{array}{c}200,500 \text { and } 200+ \\
500 \mu \mathrm{m}, \text { Cylindrical }\end{array}$ & $70 \%$ & $\begin{array}{l}\text { commercially } \\
\text { pure Ti }\end{array}$ &  & $\begin{array}{c}2.5 \times 10^{5} \\
\text { hMSCs/scaffold }\end{array}$ & 7 days \\
\hline
\end{tabular}


Table 1. Cont.

\begin{tabular}{|c|c|c|c|c|c|c|c|c|}
\hline $\begin{array}{l}\text { Author, Year, } \\
\text { Reference }\end{array}$ & Study Design & $\begin{array}{l}\text { Scaffold Size, } \\
\text { Dimension }\end{array}$ & $\begin{array}{c}\text { Pore Size, } \\
\text { Architecture }\end{array}$ & Porosity & Material & $\begin{array}{c}\text { Surface } \\
\text { Treatment }\end{array}$ & Number of Probes & Follow-Up \\
\hline $\begin{array}{l}\text { Fousová et al., } 2017 \\
\text { [89] }\end{array}$ & in vitro & $2 \mathrm{~mm}$ & $\begin{array}{l}1 \text { and } 2 \mathrm{~mm} \text {, } \\
\text { Rhombic } \\
\text { dodecahedron }\end{array}$ & - & Ti-6Al-4V & - & $\begin{array}{l}\text { 24,000 Human Bone } \\
\text { Osteosarcoma } \\
\text { Epithelial } \\
\text { Cells/scaffold }\end{array}$ & 5 days \\
\hline $\begin{array}{c}\text { Warnke et al., } 2009 \\
\text { [90] }\end{array}$ & in vitro & side length $50 \mathrm{~mm}$ & $0.45-1.2 \mathrm{~mm}$, Cubic & - & $\mathrm{Ti}_{6} \mathrm{Al}_{4} \mathrm{~V}$ & - & $\begin{array}{c}10^{5} \text { human } \\
\text { osteoblasts/scaffold }\end{array}$ & 6 weeks \\
\hline $\begin{array}{c}\text { Vaithilingam et al., } \\
2016 \text { [91] }\end{array}$ & in vitro & $\begin{array}{c}\varnothing 3 \mathrm{~mm} \times \text { height } \\
10 \mathrm{~mm} \times \text { thickness } \\
10 \mathrm{~mm}\end{array}$ & Cuboidal & - & $\mathrm{Ti}_{6} \mathrm{Al}_{4} \mathrm{~V}$ & - & $\begin{array}{l}\text { indirect study: } 800 \\
\mu L ; \text { direct study: } 80,000 \\
\text { immortalized NIH 3T3 } \\
\text { mouse embryonic } \\
\text { fibroblast cells/mL }\end{array}$ & $\begin{array}{l}\text { indirect study: } 72 \mathrm{~h} \text {; } \\
\text { direct study: } 7 \text { days }\end{array}$ \\
\hline $\begin{array}{c}\text { Matena et al., } 2015 \\
\text { [92] }\end{array}$ & in vitro & $\begin{array}{l}\text { width } 3.5 \mathrm{~mm} \times \\
\text { depth } 3.5 \mathrm{~mm} \times \\
\text { height } 1.25 \mathrm{~mm}\end{array}$ & $250 \mu \mathrm{m}$ & - & $\mathrm{TiAl}_{6} \mathrm{~V}_{4}$ & $\begin{array}{l}\text { Polycaprolactone } \\
\text { (PCL) }\end{array}$ & $\begin{array}{l}2.5 \times 10^{4} \text { Green } \\
\text { Fluorescent protein } \\
(\text { GFP)-osteoblasts/scaffolc } \\
(9 \text { scaffolds })\end{array}$ & 7 days \\
\hline $\begin{array}{c}\text { Van Bael et al., } 2012 \\
\text { [93] }\end{array}$ & in vitro & $\begin{array}{c}\varnothing 6 \mathrm{~mm} \times \text { height } \\
6 \mathrm{~mm}\end{array}$ & $\begin{array}{l}500 \text { and } 1000 \mu \mathrm{m}, \\
\text { Triangular, } \\
\text { hexagonal, } \\
\text { rectangular: }\end{array}$ & - & $\mathrm{Ti}_{6} \mathrm{Al}_{4} \mathrm{~V}$ & - & $\begin{array}{c}200,000 \\
\text { hPDC/scaffold }\end{array}$ & 14 days \\
\hline $\begin{array}{c}\text { Biemond et al., } 2013 \\
\text { [94] }\end{array}$ & in vivo & $\begin{array}{c}\varnothing 4 \mathrm{~mm} \times \text { height } \\
10 \mathrm{~mm}\end{array}$ & $\begin{array}{l}250-800 \mu \mathrm{m}, \\
\text { Cylindrical }\end{array}$ & $63 \%$ & $\mathrm{Ti}_{6} \mathrm{Al}_{4} \mathrm{~V}$ & $\begin{array}{l}\text { calcium } \\
\text { phosphate }\end{array}$ & 14 goats & 4,15 weeks \\
\hline $\begin{array}{c}\text { Pattanayak et al., } \\
2011 \text { [95] }\end{array}$ & in vivo & $\begin{array}{c}\varnothing 6 \mathrm{~mm} \times \text { height } \\
15 \mathrm{~mm}\end{array}$ & $400-800 \mu \mathrm{m}$, Cubic & $55-75 \%$ & $\mathrm{Ti}_{6} \mathrm{Al}_{4} \mathrm{~V}$ & $\begin{array}{c}\mathrm{NaOH}, \mathrm{HCl} \text { and } \\
\text { heat }\end{array}$ & 25 rabbits & $3,6,12,26,52$ weeks \\
\hline $\begin{array}{c}\text { De Wild et al., } 2013 \\
\text { [96] }\end{array}$ & in vivo & $\begin{array}{c}\varnothing 6 \text { and } 7.5 \mathrm{~mm} \times \\
\text { height } 3.8 \mathrm{~mm}\end{array}$ & $700 \mu \mathrm{m}$, Cylindrical & $83.5 \%$ & Ti-powder & $\begin{array}{l}\text { sandblasted, } \\
\text { sandblasted and } \\
\text { acid-etched }\end{array}$ & 5 rabbits & 8 weeks \\
\hline $\begin{array}{l}\text { Taniguchi et al., } \\
2015 \text { [97] }\end{array}$ & in vivo & - & $\begin{array}{l}300,600, \text { and } \\
900 \mu \mathrm{m} \text { diamond } \\
\text { crystal lattice }\end{array}$ & $65 \%$ & $\begin{array}{l}\text { pure Titanium } \\
\text { powder }\end{array}$ & - & 36 rabbits & $2,4,8$ weeks \\
\hline $\begin{array}{l}\text { Arabnejad et al., } \\
2015 \text { [98] }\end{array}$ & in vivo & - & $\begin{array}{c}500 \mu \mathrm{m} \text { and } 770 \mu \mathrm{m}, \\
\text { tetrahedron and } \\
\text { octet }\end{array}$ & $\begin{array}{l}55.51 \% \text { and } \\
69.88 \%\end{array}$ & $\mathrm{Ti}_{6} \mathrm{Al}_{4} \mathrm{~V}$ & - & 2 dogs & 4,8 weeks \\
\hline
\end{tabular}


Table 1. Cont.

\begin{tabular}{|c|c|c|c|c|c|c|c|c|}
\hline $\begin{array}{l}\text { Author, Year, } \\
\text { Reference }\end{array}$ & Study Design & $\begin{array}{l}\text { Scaffold Size, } \\
\text { Dimension }\end{array}$ & $\begin{array}{c}\text { Pore Size, } \\
\text { Architecture }\end{array}$ & Porosity & Material & $\begin{array}{c}\text { Surface } \\
\text { Treatment }\end{array}$ & Number of Probes & Follow-Up \\
\hline $\begin{array}{c}\text { Rotaru et al., } 2013 \\
\text { [99] }\end{array}$ & in vivo & $\begin{array}{c}\varnothing 5 \mathrm{~mm} \times \text { thickness } \\
1 \mathrm{~mm}\end{array}$ & Disc & $24-25 \%$ & $\mathrm{Ti}_{6} \mathrm{Al}_{7} \mathrm{Nb}$ & $\begin{array}{c}\text { hydroxyapatite } \\
\text { and } \mathrm{SiO}_{2}-\mathrm{TiO}_{2} \\
\text { solution }\end{array}$ & 36 rats & $1,2,3$ months \\
\hline $\begin{array}{c}\text { Van der Stok et al., } \\
2013[100]\end{array}$ & in vivo & $6 \mathrm{~mm}$ & $\begin{array}{c}490 \mu \mathrm{m}, \\
\text { Femur-shapoed }\end{array}$ & $\begin{array}{c}88 \% \text { and } \\
68 \%\end{array}$ & $\mathrm{Ti}_{6} \mathrm{Al}_{4} \mathrm{~V}$ & $\begin{array}{c}\text { chemical and } \\
\text { heat }\end{array}$ & 27 rats & $4,8,12$ weeks \\
\hline $\begin{array}{l}\text { Armencea et al., } \\
2015 \text { [101] }\end{array}$ & in vivo & $\begin{array}{c}\varnothing 3.3 \mathrm{~mm} \times \text { length } \\
10 \mathrm{~mm}\end{array}$ & $\begin{array}{l}\text { Cylindrical } \\
\text { screw-type }\end{array}$ & $24-25 \%$ & $\mathrm{Ti}_{6} \mathrm{Al}_{7} \mathrm{Nb}$ & $\begin{array}{l}\text { hydroxyapatite, } \\
\mathrm{SiO}_{2}-\mathrm{TiO}_{2}\end{array}$ & 18 rabbits & $1,3,6$ months \\
\hline $\begin{array}{c}\text { Peng et al., } 2016 \\
\text { [102] }\end{array}$ & in vivo & $\begin{array}{c}\varnothing 4 \mathrm{~mm} \times \text { height } \\
10 \mathrm{~mm}\end{array}$ & $\begin{array}{c}\sim 290+\sim 390 \mu \mathrm{m} \\
\text { Multi-rooted } \\
\text { implant }\end{array}$ & - & $\mathrm{Ti}_{6} \mathrm{Al}_{4} \mathrm{~V}$ & - & 33 rabbits & $4,8,12$ weeks \\
\hline $\begin{array}{l}\text { Hong Wang et al., } \\
2016 \text { [103] }\end{array}$ & $\begin{array}{l}\text { in vivo }+ \\
\text { in vitro }\end{array}$ & $\begin{array}{c}\varnothing 10 \mathrm{~mm} \times \\
\text { thickness } 2 \mathrm{~mm}\end{array}$ & Quadrate & - & $\mathrm{Ti}_{6} \mathrm{Al}_{4} \mathrm{~V}$ & - & $\begin{array}{l}4 \text { dogs, } 3 \text { rabbits, } \\
30 \text { guinea pigs }\end{array}$ & $1,3,7$ days \\
\hline $\begin{array}{l}\text { Amin Yavari et } \\
\text { al.,2014 [104] }\end{array}$ & $\begin{array}{l}\text { in vitro }+ \\
\text { in vivo }\end{array}$ & $\begin{array}{c}\varnothing 8 \mathrm{~mm} \times \text { height } 3 \\
\mathrm{~mm}\end{array}$ & $500 \mu \mathrm{m}$, Disc & $88 \%$ & $\mathrm{Ti}_{6} \mathrm{Al}_{4} \mathrm{~V}$ & $\begin{array}{c}\text { acidealkali, } \\
\text { alkalieacide } \\
\text { heat and } \\
\text { anodizing-heat }\end{array}$ & $\begin{array}{l}\text { in vitro: } 50,000 \\
\text { human } \\
\text { periosteum-derived } \\
\text { cells (hPDC)/scaffold; } \\
\text { in vivo: } 30 \text { rats }\end{array}$ & $\begin{array}{l}\text { in vitro: } 1,7,21 \\
\text { days; in vivo: } \\
12 \text { weeks }\end{array}$ \\
\hline $\begin{array}{l}\text { Jia-yun Xu et al., } \\
2016 \text { [105] }\end{array}$ & $\begin{array}{l}\text { in vitro }+ \\
\text { in vivo }\end{array}$ & $\begin{array}{l}\text { disc: } \varnothing 1 \mathrm{~mm} \times \\
\text { height } 10 \mathrm{~mm} \times \\
\text { thickness } 10 \mathrm{~mm}\end{array}$ & $600 \mu \mathrm{m}$ & - & $\begin{array}{l}\text { pure } \mathrm{Ti} \\
\text { powder }\end{array}$ & $\begin{array}{l}\text { sandblasting, } \\
\text { anodization and } \\
\text { alkali-heat }\end{array}$ & $\begin{array}{l}\text { in vitro: } 2 \times 10^{4} \\
\text { osteoblast from } \\
\text { neonatal mouse } \\
\text { calvaria; in vivo: } \\
12 \text { rabbits }\end{array}$ & $\begin{array}{c}\text { in vitro: } 1,3,5 \\
7 \text { days; in vivo: } \\
4,8 \text { weeks }\end{array}$ \\
\hline $\begin{array}{l}\text { Fukuda et al., } 2011 \\
\text { [106] }\end{array}$ & In vitro+in vivo & $\begin{array}{c}\varnothing 3.3 \mathrm{~mm} \times \text { length } \\
15 \mathrm{~mm}\end{array}$ & $\begin{array}{c}500,600,900, \text { and } \\
1200 \mu \mathrm{m}, \\
\text { Cylindrical }\end{array}$ & - & Ti powder & $\begin{array}{c}\text { Chemical and } \\
\text { heat }\end{array}$ & $\begin{array}{l}\text { in vitro: } 10 \text { samples } \\
\text { of simulated body } \\
\text { fluid; in vivo: } 8 \text { dogs }\end{array}$ & $\begin{array}{l}\text { in vitro: } 3-7 \text { days; } \\
\text { in vivo: } 16,26 \text {, } \\
\text { or } 52 \text { weeks }\end{array}$ \\
\hline
\end{tabular}


From 29 studies, 15 had an in vitro design [17,18,22,30,32,84-94], 9 in vivo [95-102], and 5 studied both in vitro and in vivo methods [32,103-106] (Figure 2). The duration of the follow-up period of the in vivo studies varies from 8 weeks to 1 year (Figure 3), while the in vitro studies vary from 5 to 52 days (Figure 4 ).

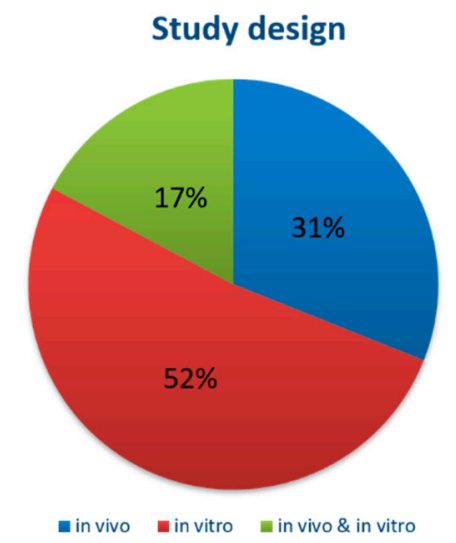

Figure 2. Synthesis of types of studies used in the review.

\section{Duration of in vivo study follow up}

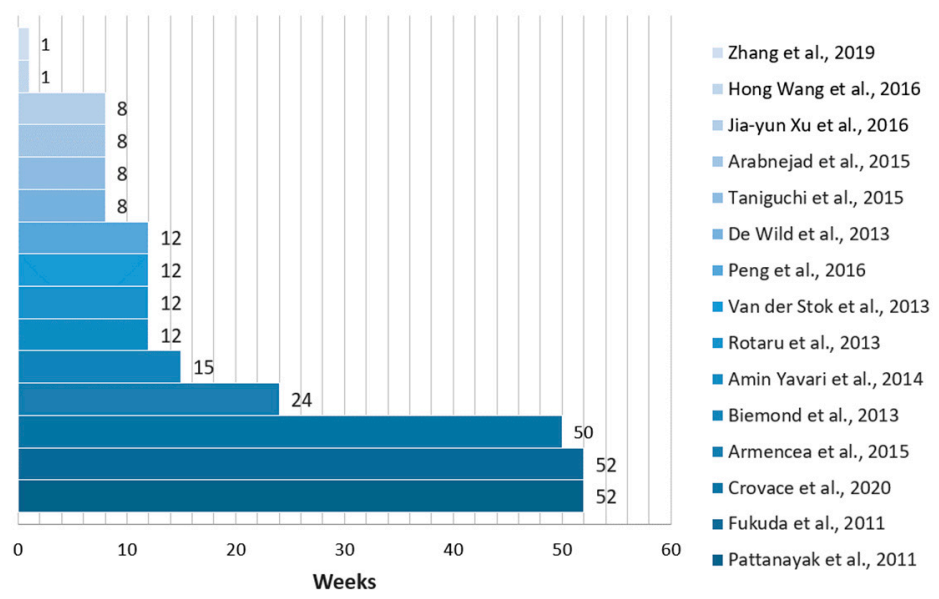

Figure 3. Duration of follow-up period of in vivo included studies.

\section{Duration of in vitro study follow up}

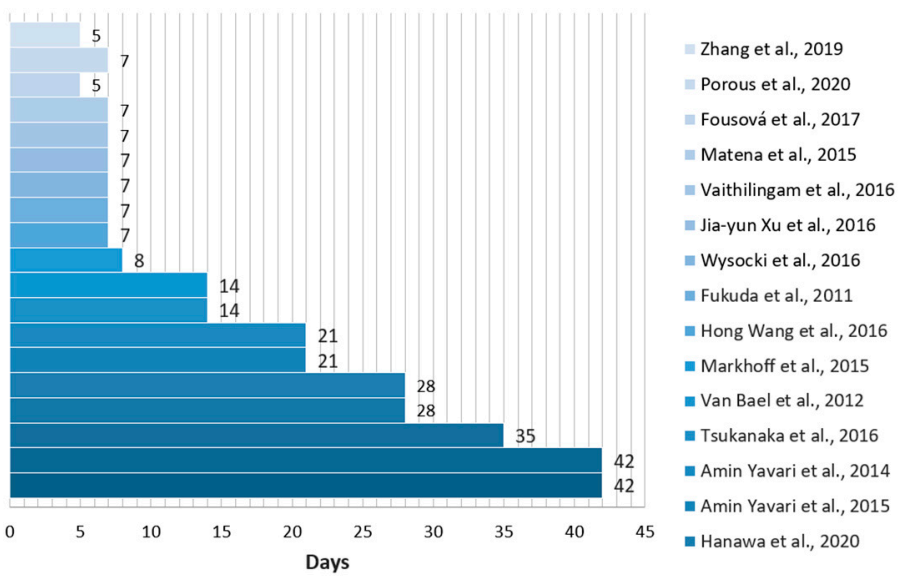

Figure 4. Duration of follow-up period of in vitro included studies. 


\subsection{Specific Characteristics of the Studies}

\subsubsection{Cytocompatibility of the SLM generated Ti Scaffolds}

All 11 in vitro and 4 combined (in vitro and in vivo) designed studies reported that SLM-generated Ti scaffolds were not cytotoxic [84-94,103-106]. Strauss et al. conducted a SEM analysis that showed the expected adipose-derived stem cells morphology that grew efficiently on the ligaments of the meshes [83]. Hong Wang et al. proved that $\mathrm{Ti}_{6} \mathrm{Al}_{4} \mathrm{~V}$ samples fabricated using SLM had good biocompatibility, matching that of the wrought group [103]. Fousová et al. confirmed in their in vitro study the cell proliferation on SLM scaffolds by assessing the metabolism between days 1 and 7. From their in vitro tests, it seemed that the material was constantly biocompatible, although microsurface chemical modifications were identified [89]. Warnke et al. also confirmed that osteoblasts cultivated directly on $\mathrm{Ti}_{6} \mathrm{Al}_{4} \mathrm{~V}$ scaffolds were highly viable. SEM evidenced that the $\mathrm{Ti}_{6} \mathrm{Al}_{4} \mathrm{~V}$ scaffold surfaces were well tolerated by osteoblasts inside the scaffolds. Osteoblasts were scattered, with attachment of filopodia to different microsurfaces' area [90]. Vaithilingam et al. sustained the previous findings, their results indicating cells' high viability [91]. In the study conducted by Matena et al., the cellular surface area increased after 7 days of cultivation, which was translated to cellular division. The results showed that pores provided a positive medium for osteoblasts regeneration [93]. All nine in vivo studies and four combined (in vitro and in vivo) proved the viability of the SLM fabricated scaffolds [94-106]. Hong Wang et al. reported that $\mathrm{SLM} \mathrm{Ti}_{6} \mathrm{Al}_{4} \mathrm{~V}$ samples did not cause dermal irritation in guinea pigs and had good blood compatibility [103]. Pattanayak et al. found no infection at the implant site and no dislocations of the implants. All implanted porous Ti metal samples were integrated to the receptor bone in all post-implantation evaluation periods. No side effects, inflammatory signs, or foreign body reactions were observed [95]. De Wild et al. observed no adverse reactions and the animals remained in good health after applying the SLM implants. In every sample, the entire volume, the bone growth volume inside the sample, and the ratio were increased at the end of the experiment [97]. In their study, Peng et al. fabricated a MRI (multi-rooted implant). The histological images of the MRIs implants were taken at 4, 8, and 12 weeks and no signs of inflammation or side effects were observed at the receptor situs-implant interfaces. During regeneration, osteoblasts matured into osteocytes resting in lacunae and embedded into the bone matrix. Primary, immature and secondary lamellar compact bones were identified at the site of new bone formation, with the bone cells dispersed on the implant surface. At 1 month evaluation, MRI showed bone marrow inside the porous structure and bone ingrowth. After 3 months, MRI pores were filled by bone tissue, with no spaces between the implants and bone tissue. After 8 weeks, substantial bone tissue extended interradiculary, and after 12 weeks, new bone tissue was identified at the root bifurcation [103].

\subsubsection{Scaffold Shape Influence on Bone Ingrowth}

From the selected 24 studies, four analyzed the influence of the scaffolds' shape on bone ingrowth (Figure 5). The in vivo study performed by Arabnejad et al. showed that bone ingrowth could be observed in both octet- and tetrahedron-shape implants at both 4 and 8 weeks. At 4 weeks, new bone formed at the implant-bone surface was identified. At 8 weeks, new bone grew inside the implant and into the nearby porous structures. The Octet truss implant showed more bone ingrowth compared with Tetrahedron implant at both time evaluations [98]. Markhoff et al. obtained the following results: Static cellular cultivation of the SLM scaffolds (cubic, pyramidal, diagonal) showed no metabolic cellular modifications after day 1 . Time positively influenced the human osteoblasts activity on the SLM scaffold with pyramidal basic structure after $96 \mathrm{~h}$ and 8 days. Contrarily, other evaluation times showed no significant differences between the two other structures. Additionally, the dynamic cultivation enhanced a superior cellular activity as well as transition through the pyramidal scaffold [86]. 


\section{Analyzed characteristics}

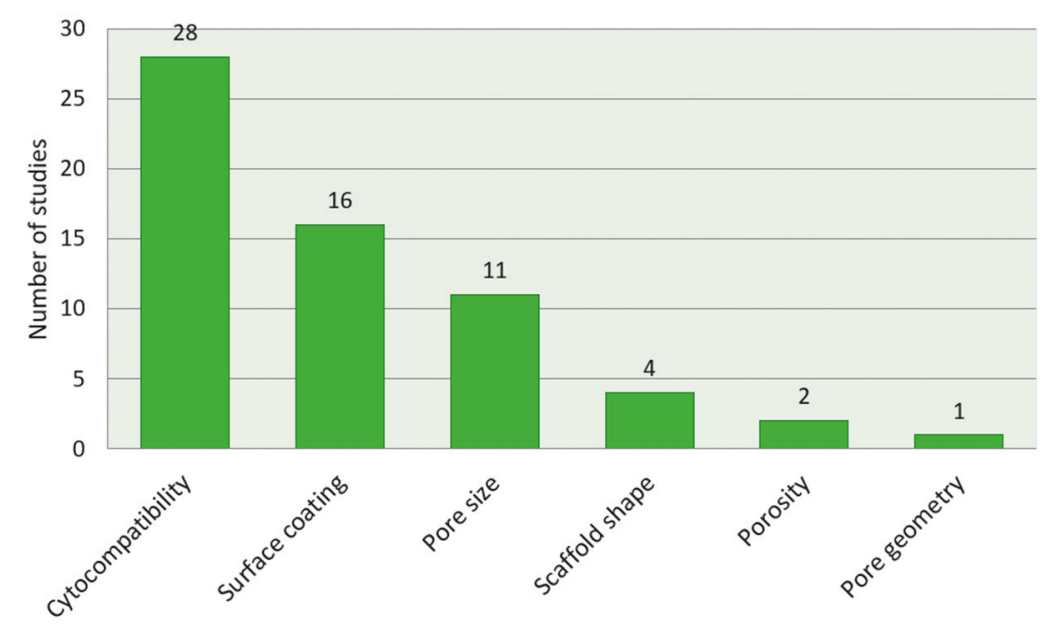

Figure 5. Synthesis of the number of studies that analysed different characteristics in the studies.

\subsubsection{Pore Size Influence on the Bone Ingrowth}

Eleven studies analyzed the influence of the pore size on the bone ingrowth (Figure 5). Five studies demonstrated that $600-1000 \mu \mathrm{m}$ pore size had a better influence on bone ingrowth (Figure 6). The in vivo study performed by Taniguchi et al. indicated that after 4 weeks, all the porosities models $(300 \mu \mathrm{m}$, $600 \mu \mathrm{m}$ and $900 \mu \mathrm{m}$ ) presented adhesion capacities after the detaching test. Bone ingrowth into the $300 \mu \mathrm{m}$ porosity implants was lower compared to the rest of the samples after 4 weeks. Due to the mechanical strength, adherence capacities, and fast bone ingrowth, the $600 \mu \mathrm{m}$ pore dimension implant proved to be adequate for orthopedic implants produced by SLM [98]. Markhoff et al. reported that the scaffold with $76 \%$ porosity and small pores $(400 \mu \mathrm{m}$ to $620 \mu \mathrm{m})$ enhanced high metabolism and ingrowth for time-related human osteoblasts and that collagen fiber production was not influenced by the scaffolds' pore size [87]. According to Fukuda et al., better osteoinduction was provided by $500 \mu \mathrm{m}$ and $600 \mu \mathrm{m}$ pore size. In their in vivo experiment, considerably more quantitative bone amount was identified for $500 \mu \mathrm{m}$ and $600 \mu \mathrm{m}$ than in $900 \mu \mathrm{m}$ and $1200 \mu \mathrm{m}$ [106]. The results of Nover et al. indicated that the apparent density, volume fraction, pore percentage, pore side length, and pore area fraction were different $(p \leq 0.001)$ for 600,900 , and $1200 \mu \mathrm{m}$ pore unit cell size groups, the $600 \mu \mathrm{m}$ pore unit cell size presenting the best results [102].

\section{Pore size}



Figure 6. Synthesis of pore size positive influence on bone ingrowth results.

In comparison, Wysocki et al. studied the $200 \mu \mathrm{m}$ and $500 \mu \mathrm{m}$ pore-sized scaffolds in vitro, their results indicating that small pores contained more numerous cells inside the scaffolds, which then negatively influenced their metabolism. This showed that in vitro cell performance could be controlled 
to a certain extent by modifying pore sizes. On the other hand, the number of viable human Mesenchimal Stem Cells (hMSCs) was significantly higher in wells used for cell seeding of scaffolds with $500 \mu \mathrm{m}$ pores than scaffolds with $200 \mu \mathrm{m}$ and bimodal pores. These results suggested that less cells were retained within scaffolds with bigger pores. The highest ALP (alkaline phosphate protein) activity per cell was measured within $500 \mu \mathrm{m}$ pore scaffolds both after 7 days of expansion and additional 7 days of osteogenic differentiation. The ALP activity was conversely correlated to total protein concentration, which suggested that the cellular differentiation was more advanced on scaffolds that supported cell proliferation to a lesser extent [102]. In the Warnke et al. study, 100\% pore occlusion was achieved at pore widths of 0.45 and $0.5 \mathrm{~mm}$ [90]. However, Matena et al. chose a small pore size of $250 \mu \mathrm{m}$ and their results indicated increased cell seeding inside the implant. The SLM produced Ti implants with $250 \mu \mathrm{m}$ pore size, which enhanced osteoblast seeding and proliferation to central pore areas [92]. Van Bael et al. compared 500 and $1000 \mu \mathrm{m}$ pore sizes and the amount of DNA measured 1 day after cell seeding showed a significantly higher DNA content for the $500 \mu \mathrm{m}$ scaffolds compared with $1000 \mu \mathrm{m}$ pore size. After 14 days of culture, the scaffolds with $1000 \mu \mathrm{m}$ scaffolds in the horizontal plane had significantly higher cell density compared with the scaffolds with a $500 \mu \mathrm{m}$ pore size in the horizontal plane for both media [93].

\subsubsection{Influence of the Porosity Degree on the Stability of the Implants}

Van der Stok et al. studied in vivo the difference between the implants with $68 \%$ and $88 \%$ porosity degree. Their results indicated that the $88 \%$ Ti structures remained completely intact in all rats, whereas breakage of some struts was seen in six of the nine rats given $68 \%$ porosity Ti implants. This was identified after 4 (two cases) or 8 weeks (four cases), but the results showed no osteointegration loss either complete loss of structural integrity of the scaffolds. The scaffolds were fixed to the proximate cortical bone and a progression of bony bridging was seen in time. The empty defect sites evidenced little bone formation and resorption of the cortical bone at the proximal and distal sites. Within the remaining defect area, high quantity of fibrous tissue was found. Histology of the Ti groups revealed formation of a major plug of new bone in the medullary canal at both ends of the bone defect. This bone most likely formed through the process of direct ossification. Bone was also abundant at the external area of the scaffolds, showing signs of an attempt to close the defect. The area inside the porous Titanium that was not filled with bone contained fibrous tissue [100]. Markhoff et al. reported that the scaffold with the highest porosity $(76 \%)$ enabled the highest metabolism and ingrowth of the human osteoblasts within time and that collagen production was not influenced by the scaffolds' pore dimension [86].

\subsubsection{Surface Coating Influence on the Properties of the SLM Implants}

Of the 29 studies, 16 analyzed the influence of coating the implants surface on inducing better mechanical strength and ingrowth characteristics (Figure 5). Enhancing bone ingrowth effect was obtained by most of the coatings and treatments (Figure 7). Biemond et al.'s results indicated that HAP (hydroxyapatite)-coated SLM specimens had significantly lower mechanical strength compared with uncoated ones, at both 4 and 15 weeks. At 1 month post-implantation, bone reaction was identified surrounding the samples. The SLM-coated surface was coated by a thick reactive tissue membrane. New woven bone was synthesized in the membrane, but no direct bone implant contact or only very limited bone ingrowth was found. The coating presented no effect on osteoconduction along the surface. After the histological and histomorphometry analysis was performed, the results showed that adding a biological coating did not significantly change the interior depth growth and sample-bone contact compared to the uncoated samples [95]. Other authors, however, obtained different results. 


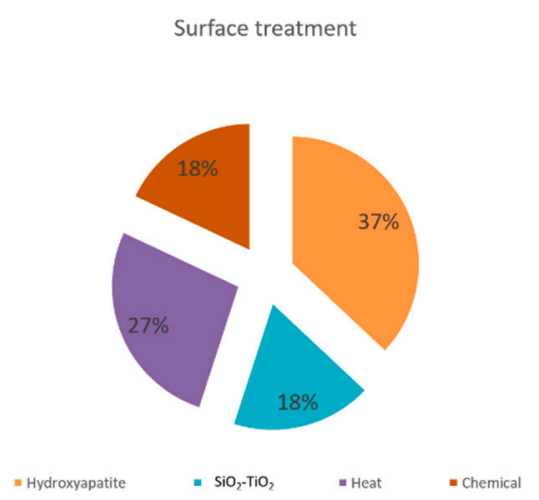

Figure 7. Synthesis of surface coating and treatment on bone ingrowth results.

Rotaru et al. studied the influence of hydroxyapatite and $\mathrm{SiO}_{2}-\mathrm{TiO}_{2}$ solution coating and proved that the Titanium alloy materials, by themselves or impregnated with $\mathrm{HAP}$ or $\mathrm{SiO}_{2}-\mathrm{TiO}_{2}$, were osteoconductive materials. One month after implantation, $\mathrm{Ti}_{6} \mathrm{Al}_{7} \mathrm{Nb}, \mathrm{Ti}_{6} \mathrm{Al}_{7} \mathrm{Nb}-\mathrm{HA}$, and $\mathrm{Ti}_{6} \mathrm{Al}_{7} \mathrm{Nb}-\mathrm{SiO}_{2}-\mathrm{TiO}_{2}$ showed bone synthesis at the margins of the samples, all with formation defects (wider defect for the third material), but none of them presented celsian signs, even the free particles of $\mathrm{SiO}_{2}-\mathrm{TiO}_{2}$ and $\mathrm{HAP}$ showed integration in the new formed bone. After 2 months, the defects in osteointegration had higher dimensions for $\mathrm{Ti}_{6} \mathrm{Al}_{7} \mathrm{Nb}$ coated with $\mathrm{SiO}_{2}-\mathrm{TiO}_{2}$, persistent for $\mathrm{Ti}_{6} \mathrm{Al}_{7} \mathrm{Nb}$ and absent for the $\mathrm{HAP}$ coating. For the 3-month period, only the alloy with $\mathrm{SiO}_{2}-\mathrm{TiO}_{2}$ coating still shows areas of integration bone loss and maybe osteolysis. In the $\mathrm{SiO}_{2}-\mathrm{TiO}_{2}$-coated implants, there were $\mathrm{SiO}_{2}-\mathrm{TiO}_{2}$ particles that detached from the implants' surface and were totally included in the newly formed bone tissue, without an inflammatory foreign body reaction. The integration of the $\mathrm{Ti}_{6} \mathrm{Al}_{7} \mathrm{Nb}$ and $\mathrm{Ti}_{6} \mathrm{Al}_{7} \mathrm{Nb}$ coated with $\mathrm{HAP}$, at 3 months, clearly shows the higher capacity of osteoinduction for these two alloys, the HAP having better histology results. Still, all of the materials showed defects of osteointegration of various degrees [99]. Similarly, Armencea et al. studied the $\mathrm{SiTiO}_{2}$ and HAP coating. After 1 month, both analyses BMD [Benchmark Dose] and histological examination) showed a high degree of bone mineralization at the implant site, similar to the results after 6 months. After 3 months, a demineralization process for the $\mathrm{Ti}_{6} \mathrm{Al}_{7} \mathrm{Nb}$ sample was observed, both when calculating the mineralized bone by histology exam and the BMD by the micro-CT exam, while the values for the coated implants are much higher [101].

Tsukanaka et al. evaluated the cellular morphology, proliferation, bone cell differentiation of the cultured osteoblasts by electron microscopy, immunofluorescent staining, proliferation assay (XTT assay), and gene expression analysis by RT-PCR (reverse transcriptase-polymerase chain reaction) after applying a bioactive treatment. The treatment stimulated osteoblast differentiation on the SLM materials [84]. Amin Yavari et al., AcA1 (applied acid-alkalibic), alkaline-acid heat treatment (AlAcH), and $\mathrm{AnH}$ (anodizing-heat treatment). Their results indicated that the largest weight percentages of $\mathrm{Ca}$ and P elements were seen in Energy-dispersive X-ray spectroscopy (EDS) analysis of AcAl surfaces. Second to AcAl surfaces were AlAcH (alkaline-acid) surfaces that showed slightly less Ca and P contents and lesser weight percentages of $\mathrm{Ca}$ and P. AnH and AsM showed the lowest apatite-forming ability with none-to-little apatite formed on the surfaces and smallest weight percentages of $\mathrm{Ca}$ and P. In terms of cell-seeding, AnH resulted in the highest efficiency; however, it was not significantly different from other surface conditions. Both AnH and AlAcH surfaces showed good cell attachments. According to the measurements of the metabolic activity, AnH showed significantly higher cell proliferation as compared to AsM specimens at 4, 7, and 21 days. They also compared sets of anodizing parameters (voltage: 10 or $20 \mathrm{~V}$ anodizing time: $30 \mathrm{~min}$ to $3 \mathrm{~h}$ ) for anodizing porous Ti structures that were later thermotreated at $500{ }^{\circ} \mathrm{C}$. Their results showed that both anodizing, and thus generation of regular nanotopographical feature, and thermal treatment had positive effect on the cell culture response to porous titanium. In particular, cell proliferation measured using metabolic activity and deoxyribonucleic acid (DNA) content was improved for anodized and thermotreatment as well as for 
anodized but not thermotreated samples. Thermotreatment upregulated the cell adsorption of porous Ti surfaces and upregulated the expression of osteogenic markers. Anodized but not thermo-treated samples showed some limited signs of upregulated expression of osteogenic markers [86]. Similarly, the results of Fukuda et al. indicated that within 16 weeks, osteoinduction was present in all the channels of all chemical and thermotreated samples $\left(5.0 \mathrm{M}\right.$ aqueous $\mathrm{NaOH}$ at $60{ }^{\circ} \mathrm{C}$ for $24 \mathrm{~h}$ and then in $0.5 \mathrm{mM} \mathrm{HCl}$ (pH 3.4) at $40{ }^{\circ} \mathrm{C}$ for $24 \mathrm{~h}$, gently washed with distilled water, and dried at $40{ }^{\circ} \mathrm{C}$ for $24 \mathrm{~h}$ at room temperature). New laminated bone was synthesized along the channel wall surface. There was no evidence of cartilaginous tissue synthesis or endochondral ossification. Contrarily, there were no signs of osteosynthesis for the un-treated implant samples for all evaluation periods, but fibro-capillary tissue was identified [106].

De Wild et al. studied the influence of sandblasting and sandblasting with acid-etched treatment of the surfaces, and obtained positive results regarding bone formation and osteoconductivity [96]. $\mathrm{Xu}$ et al. examined in vitro sandblasting and the effect of SAN (anodization) and SAH (alkali-heat treatment). Micro-/nano-textured topographies of SAN and SAH evidenced upregulated cellular metabolism correlated to the area of the surface only treated by SLA (acid etching) with significantly higher proliferation on the 1st, 3rd, 5th, and 7th day $(p<0.05)$. In vivo, SAN and SAH synthesized higher amounts of bone tissue, resulting in a larger bone-implant sample surface contact (BIC\%) and bone-bonding force than native-SLM and SLA. The alkaline phosphatase activity of SAH was significantly higher than the other three groups at an earlier stage of the 7th day ( $\mathrm{P} \mathrm{b} \mathrm{0.05),} \mathrm{and} \mathrm{the}$ $\mathrm{BIC} \%$ was nearly double that of native-SLM and SLA in the 8th week [105].

In the Wysocki et al. study, the scaffolds were chemically treated with $\mathrm{HF}$ and $\mathrm{HF}-\mathrm{HNO}_{3}$ acid solutions. The mixtures of the $\mathrm{HF} / \mathrm{HNO}_{3}$ acids were used to etch SLM samples with pore size less than $200 \mu \mathrm{m}$. The homogenous pore dimension and powder removal after chemical treatment in $\mathrm{HF} / \mathrm{HNO}_{3}$ solutions were confirmed by the Computer Tomograph (CT) evaluations and Scanning Electron Microscope (SEM) images. An additional advantage of microsurface polishing was the decrease in water contact angle by $52-75 \%$. The mechanical features of the manufactured cell structures were similar to those of human bone tissue, which might minimize the stress-shielding effect [89]. Matena et al. used PCL (polycaprolactone) coating, which decreased the amount of adhered cells compared with the non-coated Ti structure. However, the cell number regeneration and cell spreading area was unblocked by the PCL coating. Moreover, the PCL coating method kept open the structures' pores [92].

\subsubsection{Influence of Pore Geometry on Cell Proliferation}

Matena et al. studied the influence of pore geometry on the cellular viability and observed that obtuse-angled pores had a higher occlusion rate compared to acute-angled pores [92].

\section{Discussion}

The present descriptive analysis review evaluated the research evidence with a view to determining the tissue integration of the SLM-manufactured Ti scaffolds and the characteristics that influence it. We analysed a total of 24 studies that confirmed the non-cytotoxic properties of the SLM-fabricated scaffolds. However, some criteria have to be taken into account when using the implants for clinical applications. Although the various microsurface chemistry did not influence the cellular activity for the short time period in the in vitro and in vivo studies, prolonged use of the material could induce various cellular responses, as Vaithilingam et al. indicated [91]. The presence of Aluminium oxide in the oxide film may affect the stability of the oxide layer and could potentially lead to the spread of metal ions into the biological medium when inserted for a prolonged time. Also, an oxidised alloy surface will have a different reaction compared to oxidised commercially pure Ti. Due to this surface heterogeneity in different Ti samples, the selection of a suitable surface modification technique may be required, in order to form a homogenous surface and enhance the biocompatibility of the SLM-fabricated surface [92]. Peng et al. designed an MRI with 3D interconnected pore structural 
surface and different degrees of pore concentration, fabricated by SLM. The results indicated that bone can present adsorption to and cover the whole surface of the porous MRI. Histological evaluation evidenced that bone tissue penetrated into the channels of the porous MRI after insertion, and that, compared to the classical implants, the MRIs enhanced osteointegration rapidly, without changing the medium. This kind of implant could be used in clinical trials in order to provide new design characteristics that could enhance the biocompatibility of implants [102].

Regarding the influence of the scaffold's shape on the bone ingrowth, the Arabnejad et al. in vivo studies demonstrated that bone ingrowth occurs in all implants in a reproducible and predictable manner; however, in their study, the Octet truss topology proved to be a better one, by 8 weeks' time of the study, observing a further $41 \%$ and $58 \%$ increase in bone ingrowth for the Tetrahedron and Octet truss topologies [98]. Markhoff et al. conducted a static and dynamic cultivation on cells on the SLM manufactured cubic, pyramidal and diagonal structures. In both types of cultivation, the pyramidal structures showed better results [86]. Possible reasons that can be considered are porosity, pore dimension and cores' scaffold architecture. An adequate medium flow through the pyramidal structured scaffold can be assumed due to the high porosity and the open basic structure. However, there are not enough studies nor data suggesting the best shape of the SLM-fabricated structures, with the cylindrical shape being used in most of them.

Analysing the characteristics of the scaffolds is a noteworthy criteria for the success of the implantation. The pore size seems to be one of the defining features. Many authors studied different pore sizes in order to find the most appropriate one. Taniguchi et al. found out that porous Ti implants with a pore size of $600 \mu \mathrm{m}$ (P600) evidenced higher fixation capacity compared to $300 \mu \mathrm{m}$ (P300) and $900 \mu \mathrm{m}$ (P900) size, and the P300 implant evidenced lower ingrowth in trabecular bone compared with that of the P600 and P900 implants. Because of the adequate compressive strength, high adsorption capacity in the first post-implantation period, and deep bone ingrowth of the P600 implant, it represents a good candidate for the surface structure of joint prostheses, spinal fusion devices, and other individualized bone substitutes [98]. The structure, porosity and pore size of a scaffold influence cell growth behaviour. The scaffold with smaller pores (400 $\mu \mathrm{m}$ to $620 \mu \mathrm{m})$, a high porosity $(75 \%)$ and an open-porous pyramidal basic structure enabled the highest metabolic cell activity and migration and showed to be the most adequate structure, according to Markhoff et al. [86]. Similar findings were obtained by Fukuda et al., with porosity of $500 \mu \mathrm{m}$ and $600 \mu \mathrm{m}$ being more osteoinductive than $900 \mu \mathrm{m}$ and $1200 \mu \mathrm{m}$. Moreover, in $900 \mu \mathrm{m}$ and $1200 \mu \mathrm{m}$ pores, scaffolds osteoinduction was identified in the profound area of the pores [106]. Along with the physiological theory, the protected pore area in narrow or deep pores has a further advantage with respect to non-susceptibility to myogenic or fibrogenic factors from the surrounding tissue. Nover et al. came to the same conclusions in their study. Their correlation analysis indicated that the mechanical effect was higher depending on the pores dimension rather than the porosity or density [87].

A number of studies indicated that reduced-dimension pores of $250 \mu \mathrm{m}$ provided more bone ingrowth [93], whereas big pores of $1000 \mu \mathrm{m}$ were the most appropriate for implanting [94]. The differences could have multiple explanations. A significantly higher number of cells was identified for scaffolds with decreased pore dimension for day 1 after implantation. Moreover, the seeding procedure tended to be more robust when scaffolds with smaller pores were used. The two procedures could be associated to higher permeability for the samples with large pores. A higher permeability implies that the cell suspension has less resistance when permeating the scaffold, leading to higher average fluid velocities, so that the cells do not have the time to be adsorbed to the surface. Hence, the seeding is more efficient for designs with the smallest pore size. Live/dead staining and SEM visualization show substantial evidence for the cell behaviour after 14 days of in vitro culture. Independently from the pore shape, hPDC closed the pores by bridging the corners, resulting in circular cell-filled pores in all scaffolds. However, the pores with higher dimensions could be more suitable for cell proliferation/growth. The reasoning is as follows: (1) scaffolds with larger pores have more open space 
for cells to grow; and (2) a higher medium diffusivity due to an initial larger pore size and less pore occlusion, favouring oxygen and nutritive substances input.

An alternative option could be combining different pore sizes in the same scaffolds, offering bimodal implants. Wysocki et al. stated that scaffolds with smaller pores showed the highest cell adsorption, which yields the highest cell density at later time points. However, bridging of the small pores by cells might enclose the diffusion of nutrients and metabolites. Therefore, they provided the idea of bimodal scaffolds, which gives an option to associate high cell retention within the small pores with improved diffusion, and thus cells nutrition, through the large pores [89]. The same concept was proposed by Van Bael et al., based on their in vitro results. They suggested a functional graded scaffold that combines small pores for initial cell adsorption and larger non-circular pores to avoid pore occlusion over time [93]. The association of different pore sizes is an innovative tool in designing and producing scaffolds with gradient properties, which allows to mimic human bone tissue.

The porosity degree is also a property that influenced bone ingrowth. The studies conducted by Van der Stok et al. and Markhoff et al. supported the hypothesis that Ti scaffolds with higher porosity percentage provided mechanical support in the first stage post-implantation and facilitated osteogenesis over time, resulting in good mechanical strength. The high porosity $(75-88 \%)$ and open-porous pyramidal core structure proved to be the most suitable leading structure so far. Implantation of the scaffolds provides sufficient support to the defect, the capacity to assure sufficient support likely contributing to bone formation in the defects $[87,101]$.

Many studies tried to clarify which coating method or substance enhanced the osteoconductive and osteoinductive properties of the SLM fabricated scaffolds. While Biemond et al. concluded that HAP-coated SLM specimens had significantly lower mechanical strength compared to uncoated ones, and did not increase the quality of the implants behaviour [94], other authors came to different results. According to Rotaru et al. and Armencea et al., $\mathrm{Ti}_{6} \mathrm{Al}_{7} \mathrm{Nb}$ coated with $\mathrm{HAP}$ showed superior results, which could suggest a possible enhancement of osteoconductive properties by HAP infiltration. Osteointegration outcome of Ti-based implants can be improved by $\mathrm{SiO}_{2}-\mathrm{TiO}_{2}$ and $\mathrm{HAP}$ coating $[99,101]$. The HAP coating represents the best option for the $\mathrm{Ti}_{6} \mathrm{Al}_{7} \mathrm{Nb}$ alloy, which, besides the osteoinductive and osteoconductive properties (Rotaru et al.), could also induce early mineralization that supports implant osteointegration.

The influence of sandblasting and acid-etched treatment of the surfaces was demonstrated; the treated SLM surface scaffolds showed superior osteoconductivity, which facilitated more bone formation, and could be further developed for bone grafts favourable for high dimensioned defects $[95,97]$. In addition, both anodizing and thermo-treatment at $500{ }^{\circ} \mathrm{C}$ improved the cell culture activity to porous Titanium, acid etching and electropolishing chemically- and heat-treated implants being significantly greater compared to untreated implants $[96,97]$.

Moreover, the thermo-treatment seemed to improve the cell culture response to anodized specimens [62]. The addition of nano-porous characteristics on the microrough SLM Ti surface was effective in improving the bioactivity and bone regeneration [105]. The in vitro and in vivo experiments showed that surfaces modified with micro-/nano-textured hierarchical features had higher response related to cellular morphology, regeneration, differentiation, bone-implant contact and bone-bonding interaction, compared with native-SLM and SLA with microtopography only. Microscale roughness increased the mechanical stability of osteointegration, so the micro-rough surface of native SLM Ti seems to be an appropriate substrate. Micro-/nano-topography favoured the formation of a higher amount of successively regenerated bone and led to higher bone-implant contact and bone-bonding force for the in vivo bone formation. Therefore, materials fabricated by SLM had to be pre-treated with a bioactive substance to enhance the osteointegration $[85,106]$. We published two articles regarding Ti SLM in vitro and in vivo scaffolds' integration on oral cavity mesenchimal stem cells (apical papilla, interradicular bone, dental pulp) and rabbit model $[107,108]$. We used $600 \mu \mathrm{m}$ pore size with $63.4 \%$ porosity and simple Ti scaffolds coated with HA. Our results were similar to those presented in this review, that Ti scaffolds had a high biocompatibility rate and HA increased osteoblasts' proliferation. 
The animal model study showed that silicon-substituden nano-HA-coated Ti SLM scaffolds had bine regeneration in contact with hard tissue and inside the meshes' pores, being completely covered and filled with new hard tissue. This shows the importance of surface biological active substance treatment for a better osteointegration and bone regeneration. Even if one of the studies concluded that obtuse-angled pores had a better influence on cell proliferation [93], there is still no reliable evidence to confirm that pore geometry is a defining characteristic for the osteoconduction and osteoinduction of the SLM-fabricated Ti scaffolds. Besides Ti and its alloys, recent progresses in novel materials have been made, such as equiatomic, non-equiatomic Ti-Nb-Ta-Zr-Mo either Ti-15Mo-5Zr-3Al materials for biomedical purpose [109-112]. The authors showed that the implied chemical substances and their arrangement show superior mechanical properties and biocompatibility compared to $\mathrm{Ti}_{6} \mathrm{Al}_{4} \mathrm{~V}$. Moreover, the use of $\beta$-Ti alloys allows the manufacture of low Young modulus scaffolds, which are similar to bone properties [113]. The performance of this type of scaffold might be improved by thermal treatment in order to increase wettability and fibroblast surface adsorption [114]. It would be an innovative and positive approach in tissue engineering to produce equi-atomic ad crystallographic porous scaffolds of the above presented materials, which would associate the mechanical superiority to the possibility of osteoconduction and osteoinduction, in order to achieve the highest natural bone morphology and functionality. Recently, new 3D printing technologies have been developed: Laser-induced forward transfer (LIFT) for metals with high melting point; atomic diffusion additive manufacturing-ADAM (100 times faster than traditional machining, does not require post-treatment, creates complex and high quality scaffolds); nanoparticle jetting (low temperature tolerance); and binder jetting (low-cost, thermal-controlled technology, and low accuracy) [115]. These processes are trying to overcome the metal field limitations, mechanical properties and expensiveness (which limits the patients' access to treatment) of SML and EBM scaffolds. It is obvious that 3D printing technology has become important in the biomedical field, due to the patients' confidence in soft and hard tissue regenerative treatments, the increasing number of elders, and the need for individualized treatments, including orthopedic and surgical implant devices. In vitro and prospective in vivo studies are required in order to improve scaffolds' bioactive surface properties and integration.

\section{Conclusions}

Porous Ti supports bone ingrowth, which turns it into a viable bone-like base material for the manufacture of osteochondral tissue scaffolds, which represents a clinically positive option for allografts. The SLM technique used to produce the implants offers excellent control on the core structure. It is expected that by means of SLM, and a thorough research of the physiological interaction between the scaffold and bone tissue, porous bases can be created in order to efficiently modify the graft's integrative and mechanical properties in order to obtain structures able to sustain osseous tissue on Ti.

Based on the results of our descriptive review of the literature, the prognosis of SLM-fabricated Ti scaffolds is favourable. This is particularly manifest in the porous implants that have the pore dimension of 400 to $600 \mu \mathrm{m}$, have a high porosity (75-88\%), are coated with hydroxyapatite or $\mathrm{SiO}_{2}-\mathrm{TiO}_{2}$, or undergo a bioactive treatment. Studies provide both in vitro and in vivo aspects regarding SLM Ti and alloys beneficial for medical implications. Due to multiple possibilities of treating scaffolds' surface, in our opinion, future studies should focus on stimulating the regeneration of the surrounding tissue, along with the replacement of the defect. Although Ti is used as substitute for hard tissues, clinical research demonstrated that HA, calcium phosphate or BMP coating inserted into scaffolds' pores enhanced bone regeneration with superior qualities, and even inhibited malignant cells; these results might be an emergent expertise niche in which tissue engineering could associate drug delivery and biostimulative substances. The possibility of grafting hard tissue combining local hard and soft tissue treatment should be explored in prospective studies involving larger sample sizes and longer follow-up. The obtained results would represent considerable progress for both general and maxillo-facial pathologies. 
Author Contributions: Conceptualization, A.I. (Aranka Ilea) and A.-M.B.; methodology, O.S.; software, N.B.P.; validation, R.B., B.A.B., G.S. and A.I. (Aranka Ilea); formal analysis, Ş.C.V.; investigation, C.N.F.; resources, I.R.B.; data curation, A.I. (Anca Ionel); writing-original draft preparation, A.-M.B. and D.T.; writing-review and editing, B.A.B. and G.S.; visualization, A.-M.B., Ş.C.V.; supervision, A.I. (Aranka Ilea); project administration, A.I. (Aranka Ilea); funding acquisition, A.I. (Aranka Ilea). All authors have read and agreed to the published version of the manuscript.

Funding: This study was supported by the European Social Found, Human Capital Operational Program 2014-2020, project No. POCU/380/6/13/125171, a grant of the Romanian National Authority for Scientific Reasearch CNCS-UEFISCDI, project PN-III-P2-2.1-PED-2019-3664-Personalized intelligent matrices for tissue regeneration and meta-inflammation control (PRIM_TISS), No. 348PED/03.08.2020 and the internal grant No. 4995/2/08.03.2016 within the Iuliu Hațieganu University of Medicine and Pharmacy, Cluj-Napoca, Romania.

Conflicts of Interest: The authors declare no conflict of interest. The funders had no role in the design of the study; in the collection, analyses, or interpretation of data; in the writing of the manuscript, or in the decision to publish the results.

\section{References}

1. Henkel, J.; Woodruff, M.A.; Epari, D.R.; Steck, R.; Glatt, V.; Dickinson, I.C.; Choong, P.F.M.; Schuetz, M.A.; Hutmacher, D.W. Bone regeneration based on tissue engineering conceptions-A 21st century perspective. Bone Res. 2013, 13, 216-248. [CrossRef] [PubMed]

2. Jariwala, S.H.; Wee, H.; Roush, E.P.; Whitcomb, T.L.; Murter, C.; Kozlansky, G.; Lakhtakia, A.; Kunselman, A.R.; Donahue, H.J.; Armstrong, A.D.; et al. Time course of peri-implant bone regeneration around loaded and unloaded implants in a rat model. J. Orthop. Res. 2017, 35, 997-1006. [CrossRef] [PubMed]

3. Daou, E.E. Biomaterial aspects: A key factor in the longevity of implant overdenture attachment systems. J. Int. Soc. Prev. Community Dent. 2015, 5, 255-262. [CrossRef] [PubMed]

4. Prado, R.F.D.; Esteves, G.C.; Santos, E.L.D.S.; Bueno, D.A.G.; Cairo, C.A.A.; De Vasconcellos, L.G.O.; Sagnori, R.S.; Tessarin, F.B.P.; Oliveira, F.E.; De Oliveira, L.D.; et al. In vitro and in vivo biological performance of porous Ti alloys prepared by powder metallurgy. PLoS ONE 2018, 13, e0196169. [CrossRef]

5. Li, Y.; Yang, C.; Zhao, H.; Qu, S.; Li, X.; Li, Y. New developments of Ti-based alloys for biomedical applications. Materials 2014, 7, 1709-1800. [CrossRef]

6. Cohen, D.J.; Cheng, A.; Sahingur, K.; Clohessy, R.M.; Hopkins, L.B.; Boyan, B.D.; Schwartz, Z. Performance of laser sintered Ti-6Al-4V implants with bone-inspired porosity and micro/nanoscale surface roughness in the rabbit femur. Biomed. Mater. 2017, 12, 025021. [CrossRef]

7. Gao, C.; Peng, S.; Feng, P.; Shuai, C. Bone biomaterials and interactions with stem cells. Bone Res. 2017, 5, 17059. [CrossRef]

8. Jemat, A.; Ghazali, M.J.; Razali, M.; Otsuka, Y. Surface modifications and their effects on titanium dental implants. BioMed Res. Int. 2015, 2015, 791725. [CrossRef]

9. Thijs, L.; Sistiaga, M.L.M.; Wauthle, R.; Xie, Q.-G.; Kruth, J.-P.; Van Humbeeck, J. Strong morphological and crystallographic texture and resulting yield strength anisotropy in selective laser melted tantalum. Acta Mater. 2013, 61, 4657-4668. [CrossRef]

10. Wysocki, B.; Idaszek, J.; Zdunek, J.; Rożniatowski, K.; Pisarek, M.; Yamamoto, A.; Święszkowski, W. The influence of selective laser melting (SLM) process parameters on in-vitro cell response. Int. J. Mol. Sci. 2018, 19, 1619. [CrossRef]

11. Arima, Y.; Iwata, H. Effect of wettability and surface functional groups on protein adsorption and cell adhesion using well-defined mixed self-assembled monolayers. Biomaterial 2007, 28, 3074-3082. [CrossRef] [PubMed]

12. Bačáková, L.; Filová, E.; Parizek, M.; Ruml, T.; Švorčík, V. Modulation of cell adhesion, proliferation and differentiation on materials designed for body implants. Biotechnol. Adv. 2011, 29, 739-767. [CrossRef] [PubMed]

13. Xu, X.; Lu, Y.; Li, S.; Guo, S.; He, M.; Luo, K.; Lin, J. Copper-modified Ti6Al4V alloy fabricated by selective laser melting with pro-angiogenic and anti-inflammatory properties for potential guided bone regeneration applications. Mater. Sci. Eng. C 2018, 90, 198-210. [CrossRef] [PubMed]

14. Ganbold, B.; Heo, S.-J.; Koak, J.-Y.; Kim, S.-K.; Cho, J. Human stem cell responses and surface characteristics of 3D printing Co-Cr dental material. Materials 2019, 12, 3419. [CrossRef] 
15. Yuan, W.; He, X.; Zhou, X.; Zhu, Y. Hydroxyapatite nanoparticle-coated 3D-printed porous Ti6Al4V and CoCrMo alloy scaffolds and their biocompatibility to human osteoblasts. J. Nanosci. Nanotechnol. 2018, 18, 4360-4365. [CrossRef]

16. Costa, M.; Lima, R.; Melo-Fonseca, F.; Bartolomeu, F.; Alves, N.M.; Miranda, A.; Gasik, M.; Silva, F.; Silva, N.A.; Miranda, G. Development of $\beta$-TCP-Ti6Al4V structures: Driving cellular response by modulating physical and chemical properties. Mater. Sci. Eng. C 2019, 98, 705-716. [CrossRef]

17. Bartolomeu, F.; Dourado, N.; Pereira, F.; Alves, N.; Miranda, G.; Silva, F. Additive manufactured porous biomaterials targeting orthopedic implants: A suitable combination of mechanical, physical and topological properties. Mater. Sci. Eng. C 2019, 107, 110342. [CrossRef]

18. Liu, F.; Ran, Q.; Zhang, T.; Zhang, D.Z.; Su, Z. Additively Manufactured Continuous Cell-Size Gradient Porous Scaffolds: Pore Characteristics, Mechanical Properties and Biological Responses In Vitro. Materials 2020, 13, 2589. [CrossRef]

19. Ran, Q.; Yang, W.; Hu, Y.; Shen, X.; Yu, Y.; Xiang, Y.; Cai, K. Osteogenesis of 3D printed porous Ti6Al4V implants with different pore sizes. J. Mech. Behav. Biomed. Mater. 2018, 84, 1-11. [CrossRef]

20. Wally, Z.J.; Haque, A.M.; Feteira, A.; Claeyssens, F.; Goodall, R.; Reilly, G.C. Selective laser melting processed Ti6Al4V lattices with graded porosities for dental applications. J. Mech. Behav. Biomed. Mater. 2018, 90, $20-29$. [CrossRef]

21. Brunette, D.; Tenvall, P.; Textor, M.; Thomsen, P. Titanium in Medicine; Springer: Berlin, Germany, 2001.

22. Hanawa, T. Titanium-tissue interface reaction and its control with surface treatment. Front. Bioeng. Biotechnol. 2019, 7, 170. [CrossRef] [PubMed]

23. Rupp, F.; Liang, L.; Geis-Gerstorfer, J.; Scheideler, L.; Hüttig, F. Surface characteristics of dental implants: A review. Dent. Mater. 2018, 34, 40-57. [CrossRef] [PubMed]

24. Smargiassi, A.; Bertacchini, J.; Checchi, M.; Cavani, F.; Ferretti, M.; Palumbo, C. Biocompatibility analyses of Al2O3-treated titanium plates tested with osteocyte and fibroblast cell lines. Biomedicines 2017, 5, 32. [CrossRef] [PubMed]

25. Ding, R.; Wu, Z.; Qiu, G.; Wu, G.; Wang, H.; Su, X.; Yin, B.; Ma, S.; Qi, B. Selective laser sintering-produced porous titanium alloy scaffold for bone tissue engineering. Zhonghua Yi Xue Za Zhi 2014, 94, 1499-1502.

26. Bunney, P.; Zink, A.; Holm, A.; Billington, C.; Kotz, C. Orexin activation counteracts decreases in nonexercise activity thermogenesis (NEAT) caused by high-fat diet. Physiol. Behav. 2017, 176, 139-148. [CrossRef]

27. Hirota, M.; Shima, T.; Sato, I.; Ozawa, T.; Iwai, T.; Ametani, A.; Sato, M.; Noishiki, Y.; Ogawa, T.; Hayakawa, T.; et al. Development of a biointegrated mandibular reconstruction device consisting of bone compatible titanium fiber mesh scaffold. Biomaterials 2016, 75, 223-236. [CrossRef]

28. Ghosh, S.; Abanteriba, S.; Wong, S.; Houshyar, S. Selective laser melted titanium alloys for hip implant applications: Surface modification with new method of polymer grafting. J. Mech. Behav. Biomed. Mater. 2018, 87, 312-324. [CrossRef]

29. Wang, F.; Wang, L.; Feng, Y.; Yang, X.; Ma, Z.; Shi, L.; Ma, X.; Wang, J.; Ma, T.; Yang, Z.; et al. Evaluation of an artificial vertebral body fabricated by a tantalum-coated porous titanium scaffold for lumbar vertebral defect repair in rabbits. Sci. Rep. 2018, 8, 8927. [CrossRef]

30. Crovace, A.; Lacitignola, L.; Forleo, D.M.; Staffieri, F.; Francioso, E.; Di Meo, A.; Becerra, J.; Crovace, A.; Santos-Ruiz, L. 3D biomimetic porous titanium $\left(\mathrm{Ti}_{6} \mathrm{Al}_{4} \mathrm{~V}\right.$ ELI) scaffolds for large bone critical defect reconstruction: An experimental study in sheep. Animals 2020, 10, 1389. [CrossRef]

31. Major, R.; Kowalczyk, P.; Surmiak, M.; Łojszczyk, I.; Podgórski, R.; Trzaskowska, P.; Ciach, T.; Russmueller, G.; Kasperkiewicz, K.; Major, Ł.; et al. Patient specific implants for jawbone reconstruction after tumor resection. Colloids Surf. B Biointerfaces 2020, 193, 111056. [CrossRef]

32. Zhang, K.; Zhou, Y.; Xiao, C.; Zhao, W.; Wu, H.; Tang, J.; Li, Z.; Yu, S.; Li, X.; Min, L.; et al. Application of hydroxyapatite nanoparticles in tumor-associated bone segmental defect. Sci. Adv. 2019, 5, eaax6946. [CrossRef] [PubMed]

33. Maroulakos, M.; Kamperos, G.; Tayebi, L.; Halazonetis, D.; Ren, Y. Applications of 3D printing on craniofacial bone repair: A systematic review. J. Dent. 2019, 80, 1-14. [CrossRef] [PubMed]

34. Qi, J.; Zhang, Y.; Liu, X.; Zhang, Q.; Xiong, C. Preparation and properties of a biodegradable poly(lactide-co-glycolide)/poly(trimethylene carbonate) porous composite scaffold for bone tissue engineering. New J Chem 2020, 44, 14632-14641. [CrossRef] 
35. Simon, M.; Lagneau, C.; Moreno, J.; Lissac, M.; Dalard, F.; Grosgogeat, B. Corrosion resistance and biocompatibility of a new porous surface for titanium implants. Eur. J. Oral Sci. 2005, 113, 537-545. [CrossRef]

36. Fouda, M.F.A.; Nemat, A.; Gawish, A.; Baiuomy, A.R. Does the coating of titanium implants by hydroxyapatite affect the elaboration of free radicals. An experimental study. Aust. J. Basic Appl. Sci. 2009, 3, 1122-1129.

37. Simmons, C.A.; Valiquette, N.; Pilliar, R.M. Osseointegration of sintered porous-surfaced and plasma spray-coated implants: An animal model study of early postimplantation healing response and mechanical stability. J. Biomed. Mater. Res. 1999, 47, 127-138. [CrossRef]

38. Cho, S.-B.; Nakanishi, K.; Kokubo, T.; Soga, N.; Ohtsuki, C.; Nakamura, T.; Kitsugi, T.; Yamamuro, T. Dependence of apatite formation on silica gel on its structure: Effect of heat treatment. J. Am. Ceram. Soc. 1995, 78, 1769-1774. [CrossRef]

39. Xue, W.; Liu, X.; Zheng, X.; Ding, C. In vivo evaluation of plasma-sprayed titanium coating after alkali modification. Biomaterial 2005, 26, 3029-3037. [CrossRef]

40. Knabe, C.; Klar, F.; Fitzner, R.; Radlanski, R.; Gross, U. In vitro investigation of titanium and hydroxyapatite dental implant surfaces using a rat bone marrow stromal cell culture system. Biomaterial 2002, 23, 3235-3245. [CrossRef]

41. Guo, L.; Wu, H.; Liu, X.; Zhu, Y.; Gao, J.; Guo, T. Effect of fluoride corrosion on the bonding strenght of Ti-porcelain under statis loads. Mater. Lett. 2009, 63, 2486-2488. [CrossRef]

42. Katic, V.; Curkovic, L.; Bosnjak, M.U.; Peros, K.; Mandic, D.; Spalj, S. Effect of pH, fluoride and hydrofluoric acid concentration on ion release from NiTi wires with various coatings. Dent. Mater. J. 2017, 36, 149-156. [CrossRef] [PubMed]

43. Wang, X.; Bai, S.; Li, F.; Li, N.; Zhang, J.; Tian, M.; Zhang, Q.; Tong, Y.; Zhang, Z.; Wang, G.; et al. Effect of plasma nitriding and titanium nitride coating on the corrosion resistance of titanium. J. Prosthet. Dent. 2016, 116, 450-456. [CrossRef] [PubMed]

44. Tamaki, Y.; Miyazaki, T.; Suzuki, E.; Miyaji, T. Polishing of titanium prosthetics (Part 6). The chemical polishing baths containing hydrofluoric acid and nitric acid. Shika Zair. Kikai 1989, 8, 103-109.

45. Wysocki, B.; Idaszek, J.; Buhagiar, J.; Szlązak, K.; Brynk, T.; Kurzydłowski, K.J.; Święszkowski, W. The influence of chemical polishing of titanium scaffolds on their mechanical strength and in-vitro cell response. Mater. Sci. Eng. C Mater. Boil. Appl. 2018, 95, 428-439. [CrossRef]

46. Ong, J.L.; Chan, D.C.N. Hydroxyapatite and their use as coatings in dental implants: A review. Crit. Rev. Biomed. Eng. 2000, 28, 667-707. [CrossRef]

47. Le Guehennec, L.; Soueidan, A.; Layrolle, P.; Amouriq, Y. Surface treatments of titanium dental implants for rapid osseointegration. Dent. Mater. 2007, 23, 844-854. [CrossRef]

48. Boyan, B.D.; Cheng, A.; Olivares-Navarrete, R.; Schwartz, Z. Implant Surface design regulates mesenchymal stem cell differentiation and maturation. Adv. Dent. Res. 2016, 28, 10-17. [CrossRef]

49. Lewallen, E.A.; Jones, D.L.; Dudakovic, A.; Thaler, R.; Paradise, C.R.; Kremers, H.M.; Abdel, M.P.; Kakar, S.; Dietz, A.B.; Cohen, R.C.; et al. Osteogenic potential of human adipose-tissue-derived mesenchymal stromal cells cultured on 3D-printed porous structured titanium. Gene 2016, 581, 95-106. [CrossRef]

50. Xu, Y.; Zhang, D.; Zhou, Y.; Wang, W.; Cao, X. Study on topology optimization design, manufacturability, and performance evaluation of Ti-6Al-4V porous structures fabricated by selective laser melting (SLM). Materials 2017, 10, 1048.

51. Attar, H.; Calin, M.; Zhang, L.C.; Scudino, S.; Eckert, J. Manufacture by selective laser melting and mechanical behavior of commercially pure titanium. Mat. Sci. Eng. 2014, 21, 170-177. [CrossRef]

52. Lewandowski, J.J.; Seifi, M. Metal additive manufacturing: A review of mechanical properties. Annu. Rev. Mater. Res. 2016, 46, 151-186. [CrossRef]

53. Hrabe, N.W.; Gnäupel-Herold, T.; Quinn, T. Fatigue properties of a titanium alloy (Ti-6Al-4V) fabricated via electron beam melting (EBM): Effects of internal defects and residual stress. Int. J. Fatigue 2017, 94, 202-210. [CrossRef]

54. Prashanth, K.G.; Kolla, S.; Eckert, J. Additive Manufacturing Processes: Selective Laser Melting, Electron Beam Melting and Binder Jetting-Selection Guidelines. Materials 2017, 10, 672. [CrossRef]

55. Prashanth, K.G.; Scudino, S.; Eckert, J.; Prashanth, K.G. Defining the tensile properties of Al-12Si parts produced by selective laser melting. Acta Mater. 2017, 126, 25-35. [CrossRef] 
56. Trevisan, F.; Calignano, F.; Aversa, A.; Marchese, G.; Lombardi, M.; Biamino, S.; Ugues, D.; Manfredi, D. Additive manufacturing of titanium alloys in the biomedical field: Processes, properties and applications. J. Appl. Biomater. Funct. Mater. 2017, 16, 57-67. [CrossRef]

57. Bormann, T.; Schumacher, R.; Müller, B.; Mertmann, M.; De Wild, M. Tailoring selective laser melting process parameters for NiTi implants. J. Mater. Eng. Perform. 2012, 21, 2519-2524. [CrossRef]

58. Šittner, P.; Heller, L.; Pilch, J.; Curfs, C.; Alonso, T.; Favier, D.; Alonso, T. Young's modulus of austenite and martensite phases in superelastic NiTi wires. J. Mater. Eng. Perform. 2014, 23, 2303-2314. [CrossRef]

59. Vaithilingam, J.; Kilsby, S.; Goodridge, R.; Christie, S.D.; Edmondson, S.; Hague, R.J. Functionalization of Ti6Al4V components fabricated using selective laser melting with a bioactive compound. Mater. Sci. Eng. C Mater. Biol. Appl. 2015, 46, 52-61. [CrossRef]

60. Sing, S.L.; An, J.; Yeong, W.Y.; Wiria, F.E. Laser and electron-beam powder-bed additive manufacturing of metallic implants: A review on processes, materials and designs. J. Orthop. Res. 2015, 34, 369-385. [CrossRef]

61. Ponader, S.; Vairaktaris, E.; Heinl, P.; Wilmowsky, C.V.; Rottmair, A.; Körner, C.; Singer, R.F.; Holst, S.; Schlegel, K.A.; Neukam, F.W.; et al. Effects of topographical surface modifications of electron beam melted Ti-6Al-4V titanium on human fetal osteoblasts. J. Biomed. Mater. Res. Part A 2008, 84, 1111-1119. [CrossRef]

62. Ou, S.-F.; Peng, B.-Y.; Chen, Y.-C.; Tsai, M.-H. Manufacturing and characterization of NiTi alloy with functional properties by selective laser melting. Metals 2018, 8, 342. [CrossRef]

63. Yuan, B.; Zhu, M.; Chung, C. Biomedical Porous shape memory alloys for hard-tissue replacement materials. Materials 2018, 11, 1716. [CrossRef] [PubMed]

64. Bormann, T.; Friess, S.; De Wild, M.; Schumacher, R.; Schulz, G.; Müller, B. Determination of strain fields in porous shape memory alloys using micro-computed tomography. In Developments in X-ray Tomography VII; Stock, S.R., Ed.; SPIE Digital Library: Bellingham, WA, USA, 2010; Volume 7804. [CrossRef]

65. Sabahi, N.; Chen, W.; Wang, C.-H.; Kruzic, J.J.; Li, X. A Review on additive manufacturing of shape-memory materials for biomedical applications. JOM 2020, 72, 1229-1253. [CrossRef]

66. Wang, X.; Kustov, S.; Van Humbeeck, J. A Short Review on the microstructure, transformation behavior and functional properties of NiTi shape memory alloys fabricated by selective laser melting. Materials 2018, 11, 1683. [CrossRef] [PubMed]

67. Khoo, Z.X.; Liu, Y.; An, J.; Chua, C.K.; Shen, Y.-F.; Kuo, C.-N. A review of selective laser melted NiTi Shape memory alloy. Materials 2018, 11, 519. [CrossRef]

68. Bansiddhi, A.; Sargeant, T.; Stupp, S.; Dunand, D.C. Porous NiTi for bone implants: A review. Acta Biomater. 2008, 4, 773-782. [CrossRef]

69. Zhang, Y.; Li, D.; Zhang, X.P. Gradient porosity and large pore size NiTi shape memory alloys. Scr. Mater. 2007, 57, 1020-1023. [CrossRef]

70. Es-Souni, M.; Es-Souni, M.; Fischer-Brandies, H. Assessing the biocompatibility of NiTi shape memory alloys used for medical applications. Anal. Bioanal. Chem. 2005, 381, 557-567. [CrossRef]

71. Saedi, S.; Saghaian, S.E.; Jahadakbar, A.; Moghaddam, N.S.; Andani, M.T.; Lu, Y.C.; Elahinia, M.; E Karaca, H.; Saghaian, S.M. Shape memory response of porous NiTi shape memory alloys fabricated by selective laser melting. J. Mater. Sci. Mater. Electron. 2018, 29, 40. [CrossRef]

72. Bassani, P.; Panseri, S.; Ruffini, A.; Montesi, M.; Ghetti, M.; Zanotti, C.; Tampieri, A.; Tuissi, A. Porous NiTi shape memory alloys produced by SHS: Microstructure and biocompatibility in comparison with $\mathrm{Ti}_{2} \mathrm{Ni}$ and $\mathrm{TiNi}_{3}$. J. Mater. Sci. Mater. Electron. 2014, 25, 2277-2285. [CrossRef]

73. Sillberstei, B.; Gyunther, V. Shape-memory implants in spinal surgery: Long-term results. In Shape Memory Implants; Springer: Berlin, Germany, 2000; pp. 147-152.

74. Shishkovsky, A. Porous biocompatible implants and tissue scaffolds synthesized by selective laser sintering fron Ti and NiTi. J. Mster. Chem. 2008, 18, 1309-1317. [CrossRef]

75. Xing, H.; Li, R.; Wei, Y.; Ying, B.; Li, D.; Qin, Y. Improved osteogenesis of selective-laser-melted titanium alloy by coating strontium-doped phosphate with high-efficiency air-plasma treatment. Front. Bioeng. Biotechnol. 2020, 8. [CrossRef] [PubMed]

76. .Coskun, M.E.; Coskun, K.A.; Tutar, Y. Determination of Optimum Operation Parameters for Low-Intensity Pulsed Ultrasound and Low-Level Laser Based Treatment to Induce Proliferation of Osteoblast and Fibroblast Cells. Photomed Laser Surg 2018, 36, 246-252. [CrossRef] [PubMed] 
77. Alcade, M.P.; Duarte, M.A.H.; Vasconcelos, B.C.; Tanomaru-Filho, M.; Vasconcelos, B.C.; Só, M.V.R.; Vivan, R.R. Torsional fatigue resistance of pathfinding instruments manufactured from several NiTi alloys. Int. Endod. 2018, 51, 697-704. [CrossRef]

78. Zupanc, J.; Vahdat-Pajouh, N.; Schäfer, E. New thermomechanically treated NiTi alloys-A review. Int. Endod. J. 2018, 51, 1088-1103. [CrossRef]

79. Pereira, E.S.J.; Peixoto, I.F.C.; Viana, A.C.D.; Oliveira, I.I.; Gonzalez, B.M.; Buono, V.T.L.; Bahia, M.G.D.A. Physical and mechanical properties of a thermomechanically treated NiTi wire used in the manufacture of rotary endodontic instruments. Int. Endod. J. 2011, 45, 469-474. [CrossRef]

80. Sallica-Leva, E.; Jardini, A.; Fogagnolo, J.B. Microstructure and mechanical behavior of porous Ti-6Al-4V parts obtained by selective laser melting. J. Mech. Behav. Biomed. Mater. 2013, 26, 98-108. [CrossRef]

81. Olakanmi, E.O.; Cochrane, R.; Dalgarno, K. A review on selective laser sintering/melting (SLS/SLM) of aluminium alloy powders: Processing, microstructure, and properties. Prog. Mater. Sci. 2015, 74, 401-477. [CrossRef]

82. Moher, D.; Liberati, A.; Tetzlaff, J.; Altman, D.G.; Group, T.P. Preferred reporting items for systematic reviews and meta-analyses: The PRISMA statement. PLoS Med. 2009, 6, e1000097. [CrossRef]

83. Strauß, S.; Dudziak, S.; Hagemann, R.; Barcikowski, S.; Fliess, M.; Israelowitz, M.; Kracht, D.; Kuhbier, J.W.; Radtke, C.; Reimers, K.; et al. Induction of osteogenic differentiation of adipose derived stem cells by microstructured nitinol actuator-mediated mechanical stress. PLoS ONE 2012, 7, e51264. [CrossRef]

84. Tsukanaka, M.; Fujibayashi, S.; Takemoto, M.; Matsushita, T.; Kokubo, T.; Nakamura, T.; Sasaki, K.; Matsuda, S. Bioactive treatment promotes osteoblast differentiation on titanium materials fabricated by selective laser melting technology. Dent. Mater. J. 2016, 35, 118-125. [CrossRef] [PubMed]

85. Yavari, S.A.; Chai, Y.; Böttger, A.J.; Wauthle, R.; Schrooten, J.; Weinans, H.; Zadpoor, A.A. Effects of anodizing parameters and heat treatment on nanotopographical features, bioactivity, and cell culture response of additively manufactured porous titanium. Mater. Sci. Eng. C 2015, 51, 132-138. [CrossRef] [PubMed]

86. Markhoff, J.; Wieding, J.; Weißmann, V.; Pasold, J.; Jonitz, A.; Bader, R. Influence of different three-dimensional open porous titanium scaffold designs on human osteoblasts behavior in static and dynamic cell investigations. Materials 2015, 8, 5490-5507. [CrossRef] [PubMed]

87. Nover, A.B.; Lee, S.L.; Georgescu, M.S.; Howard, D.R.; Saunders, R.A.; Yu, W.T.; Klein, R.W.; Napolitano, A.P.; Ateshian, G.A.; Hung, C.T. Porous titanium bases for osteochondral tissue engineering. Acta Biomater. 2015, 27, 286-293. [CrossRef]

88. Wysocki, B.; Idaszek, J.; Szlązak, K.; Strzelczyk, K.; Brynk, T.; Kurzydłowski, K.J.; Święszkowski, W. Post processing and biological evaluation of the titanium scaffolds for bone tissue engineering. Materials 2016, 9, 197. [CrossRef]

89. Fousova, M.; Vojtěch, D.; KUBÁSEK, J.; Jablonska, E.; Fojt, J. Promising characteristics of gradient porosity Ti-6Al-4V alloy prepared by SLM process. J. Mech. Behav. Biomed. Mater. 2017, 69, 368-376. [CrossRef]

90. Warnke, P.H.; Douglas, T.E.L.; Wollny, P.; Sherry, E.; Steiner, M.; Galonska, S.; Becker, S.T.; Springer, I.N.; Wiltfang, J.; Sivananthan, S. Rapid prototyping: Porous titanium alloy scaffolds produced by selective laser melting for bone tissue engineering. Tissue Eng. Part C Methods 2009, 15, 115-124. [CrossRef]

91. Vaithilingam, J.; Prina, E.; Goodridge, R.; Hague, R.J.; Edmondson, S.; Rose, F.R.A.J.; Christie, S.D.R. Surface chemistry of Ti6Al4V components fabricated using selective laser melting for biomedical applications. Mater. Sci. Eng. C 2016, 67, 294-303. [CrossRef]

92. Matena, J.; Petersen, S.; Gieseke, M.; Kampmann, A.; Teske, M.; Beyerbach, M.; Escobar, H.M.; Haferkamp, H.; Gellrich, N.-C.; Nolte, I. SLM produced porous titanium implant improvements for enhanced vascularization and osteoblast seeding. Int. J. Mol. Sci. 2015, 16, 7478-7492. [CrossRef]

93. Van Bael, S.; Chai, Y.; Truscello, S.; Moesen, M.; Kerckhofs, G.; Van Oosterwyck, H.; Kruth, J.-P.; Schrooten, J. The effect of pore geometry on the in vitro biological behavior of human periosteum-derived cells seeded on selective laser-melted Ti6Al4V bone scaffolds. Acta Biomater. 2012, 8, 2824-2834. [CrossRef]

94. Biemond, J.E.; Hannink, G.; Verdonschot, N.; Buma, P. Bone ingrowth potential of electron beam and selective laser melting produced trabecular-like implant surfaces with and without a biomimetic coating. J. Mater. Sci. Mater. Electron. 2012, 24, 745-753. [CrossRef] [PubMed]

95. Pattanayak, D.K.; Fukuda, A.; Matsushita, T.; Takemoto, M.; Fujibayashi, S.; Sasaki, K.; Nishida, N.; Nakamura, T.; Kokubo, T. Bioactive Ti metal analogous to human cancellous bone: Fabrication by selective laser melting and chemical treatments. Acta Biomater. 2011, 7, 1398-1406. [CrossRef] [PubMed] 
96. De Wild, M.; Schumacher, R.; Mayer, K.; Schkommodau, E.; Thoma, D.S.; Bredell, M.; Gujer, A.K.; Grätz, K.W.; Weber, F.E. Bone regeneration by the osteoconductivity of porous titanium implants manufactured by selective laser melting: A histological and micro computed tomography study in the rabbit. Tissue Eng. Part A 2013, 19, 2645-2654. [CrossRef] [PubMed]

97. Taniguchi, N.; Fujibayashi, S.; Takemoto, M.; Sasaki, K.; Otsuki, B.; Nakamura, T.; Matsushita, T.; Kokubo, T.; Matsuda, S. Effect of pore size on bone ingrowth into porous titanium implants fabricated by additive manufacturing: An in vivo experiment. Mater. Sci. Eng. C 2016, 59, 690-701. [CrossRef]

98. Arabnejad, S.; Johnston, R.B.; Pura, J.A.; Singh, B.; Tanzer, M.; Pasini, D. High-strength porous biomaterials for bone replacement: A strategy to assess the interplay between cell morphology, mechanical properties, bone ingrowth and manufacturing constraints. Acta Biomater. 2016, 30, 345-356. [CrossRef]

99. Rotaru, H.; Armencea, G.; Spîrchez, D.; Berce, C.; Marcu, T.; Leordean, D.; Kim, S.G.; Dinu, C.; Băciuț, G.; Băciut, M. In vivo behavior of surface modified Ti6Al7Nb alloys used in selective laser melting for custom-made implants. A preliminary study. Rom. J. Morphol. Embryol. 2013, 54, 791-796.

100. Van Der Stok, J.; Van Der Jagt, O.P.; Yavari, S.A.; De Haas, M.F.P.; Waarsing, J.H.; Jahr, H.; Van Lieshout, E.M.; Patka, P.; Verhaar, J.A.N.; Zadpoor, A.A.; et al. Selective laser melting-produced porous titanium scaffolds regenerate bone in critical size cortical bone defects. J. Orthop. Res. 2012, 31, 792-799. [CrossRef]

101. Armencea, G.; Berce, C.; Rotaru, H.; Bran, S.; Leordean, D.; Coada, C.; Todea, M.; Jula, C.A.; Gheban, D.; Băciuţ, G.; et al. Micro-CT and histological analysis of Ti6Al7Nb custom made implants with hydroxyapatite and $\mathrm{SiO} 2-\mathrm{TiO} 2$ coatings in a rabbit model. Clujul Med. 2015, 88, 408-414. [CrossRef]

102. Peng, W.; Xu, L.-W.; You, J.; Fang, L.; Zhang, Q. Selective laser melting of titanium alloy enables osseointegration of porous multi-rooted implants in a rabbit model. Biomed. Eng. Online 2016, 15, 85. [CrossRef]

103. Wang, H.; Zhao, B.; Liu, C.; Wang, C.; Tan, X.; Hu, M. A Comparison of Biocompatibility of a Titanium Alloy Fabricated by Electron Beam Melting and Selective Laser Melting. PLoS ONE 2016, 11, e0158513. [CrossRef]

104. Yavari, S.A.; Van Der Stok, J.; Chai, Y.C.; Wauthle, R.; Birgani, Z.T.; Habibović, P.; Mulier, M.; Schrooten, J.; Weinans, H.; Zadpoor, A.A. Bone regeneration performance of surface-treated porous titanium. Biomaterial 2014, 35, 6172-6181. [CrossRef]

105. Xu, J.-Y.; Chen, X.-S.; Zhang, C.-Y.; Liu, Y.; Wang, J.; Deng, F.-L. Improved bioactivity of selective laser melting titanium: Surface modification with micro-/nano-textured hierarchical topography and bone regeneration performance evaluation. Mater. Sci. Eng. C Mater. Biol. Appl. 2016, 68, 229-240. [CrossRef] [PubMed]

106. Fukuda, A.; Takemoto, M.; Saito, T.; Fujibayashi, S.; Neo, M.; Pattanayak, D.K.; Matsushita, T.; Sasaki, K.; Nishida, N.; Kokubo, T.; et al. Osteoinduction of porous Ti implants with a channel structure fabricated by selective laser melting. Acta Biomater. 2011, 7, 2327-2336. [CrossRef] [PubMed]

107. Ilea, A.; Timuş, D.; Petrescu, N.B.; SoriŢău, O.; Boşca, B.A.; Mager, V.; Barbu-Tudoran, L.; Băbţan, A.M.; Câmpian, R.S.; Barabás, R. An in vitro Study on the biocompatibility of titanium implants made by selective laser melting. Biotechnol. Bioprocess Eng. 2019, 24, 782-792. [CrossRef]

108. Ilea, A.; Vrabie, O.-G.; Băbțan, A.-M.; Miclăuş, V.; Ruxanda, F.; Sárközi, M.; Barbu-Tudoran, L.; Mager, V.; Berce, C.; Boșca, A.B.; et al. Osseointegration of titanium scaffolds manufactured by selective laser melting in rabbit femur defect model. J. Mater. Sci. Mater. Med. 2019, 30, 26. [CrossRef] [PubMed]

109. Ishimoto, T.; Hagihara, K.; Hisamoto, K.; Sun, S.-H.; Nakano, T. Crystallographic texture control of beta-type Ti-15Mo-5Zr-3Al alloy by selective laser melting for the development of novel implants with a biocompatible low Young's modulus. Scr. Mater. 2017, 132, 34-38. [CrossRef]

110. Hori, T.; Nagase, T.; Todai, M.; Matsugaki, A.; Nakano, T. Development of non-equiatomic Ti-Nb-Ta-Zr-Mo high-entropy alloys for metallic biomaterials. Scr. Mater. 2019, 172, 83-87. [CrossRef]

111. Nagase, T.; Todai, M.; Hori, T.; Nakano, T. Microstructure of equiatomic and non-equiatomic Ti-Nb-Ta-Zr-Mo high-entropy alloys for metallic biomaterials. J. Alloys Compd. 2018, 753, 412-421. [CrossRef]

112. Rondelli, G.; Vicentini, B. Evaluation by electrochemical tests of the passive film stability of equiatomic Ni-Ti alloy also in presence of stress-induced martensite. J. Biomed. Mater. Res. 2000, 51, 47-54. [CrossRef]

113. Nagase, T.; Hori, T.; Todai, M.; Sun, S.-H.; Nakano, T. Additive manufacturing of dense components in beta-titanium alloys with crystallographic texture from a mixture of pure metallic element powders. Mater. Des. 2019, 173, 107771. [CrossRef] 
114. Toffoli, A.; Parisi, L.; Bianchi, M.G.; Lumetti, S.; Bussolati, O.; Macaluso, G.M. Thermal treatment to increase titanium wettability induces selective proteins adsorption from blood serum thus affecting osteoblasts adhesion. Mater. Sci. Eng. C 2019, 107, 110250. [CrossRef] [PubMed]

115. Ni, J.; Ling, H.; Zhang, S.; Wang, Z.; Peng, Z.; Benyshek, C.; Zan, R.; Miri, A.K.; Li, Z.; Zhang, X.; et al. Three-dimensional printing of metals for biomedical applications. Mater. Today BIO 2019, 3, 100024. [CrossRef] [PubMed]

(C) 2020 by the authors. Licensee MDPI, Basel, Switzerland. This article is an open access article distributed under the terms and conditions of the Creative Commons Attribution (CC BY) license (http://creativecommons.org/licenses/by/4.0/). 